\title{
Exploration of Financial Market Credit Scoring and Risk Management and Prediction Using Deep Learning and Bionic Algorithm
}

Peng Du, School of Economics and Management, Shaanxi University of Science and Technology, China*

Hong Shu, Department of Public Courses, Shaanxi Polytechnic Institute, China

\begin{abstract}
The purpose is to effectively manage the financial market, comprehensively assess personal credit, and reduce the risk of financial enterprises. Given the systemic risk problem caused by the lack of credit scoring in the existing financial market, a credit scoring model is put forward based on the deep learning network. The proposed model uses RNN (recurrent neural network) and BRNN (bidirectional recurrent neural network) to avoid the limitations of shallow models. Afterward, to optimize path analysis, bionic optimization algorithms are introduced, and an integrated deep learning model is proposed. Finally, a financial credit risk management system using the integrated deep learning model is proposed. The probability of default or overdue customers is predicted through verification on three real credit data sets, thus realizing the credit risk management for credit customers.
\end{abstract}

\section{KEYWORDS}

Bionic Algorithm, Deep Learning, Financial Market, Integrated Model, Risk Management

\section{INTRODUCTION}

The collapse of the Bretton Woods system has created a lasting influence on international financial markets that have become extremely volatile under accelerating economic globalization (Nikulin \& Pekhterev, 2021). In such a financial environment, enterprises, financial institutions, and individual investors might have to bear various unprecedented risks (Nosan, 2019), which seriously harms the healthy development of the national economy and global financial markets (Olivier \& Lieven, 2019). In particular, the 2008 subprime mortgage crisis has featured the bankruptcy of numerous enterprises and huge losses of financial institutions (Soares et al., 2021). Worse still, without a global financial regulation system, the international financial market is becoming ever more complicated (Thomas, 2017). Generally, enterprises, financial institutions, and individual investors utilize effective risk prediction models to analyze financial data and avert risks (Ouyang et al., 2021). Compared with manual analysis, model prediction is more objective and can interpret financial data from multiple angles, avoiding potential systematic risks (Zhou, 2017). Therefore, the study of the financial market risk management system plays an important role in ensuring the stability of the national financial market. 
Initially, the variance-based risk management system is used for the financial market, which considers the return surplus over the average into risk management and takes the average return as the benchmark (Sun, 2021); although the average deviation is considered in the model, it is not suitable to predict small probability events (Ma et al., 2020). Later, the Value at Risk (VaR) model comes into being, which can effectively control the portfolio risks under the same number of securities (Elena, 2019); still, this model is unstable and highly dependent on product types (Hee \& Christian, 2021). Afterward, the Coherentmeasure of Risk (COR) model is proposed, which has shown great practical significance in the determination of capital and portfolio of banks (Veryzhenko, 2021); yet, this model has presented low-linearization ability because of high dimensionality and complex calculation (Dolfin, 2019). Recently, a new risk management model is proposed based on deep learning. Deep learning learns the inherent laws and representation features of sample data and uses them to interpret text, image, and sound data. Deep learning aims to intellectualize machines with the same analytical and learning ability as humans to recognize various data (Pang et al., 2019), which is a complex machine learning algorithm and is extremely effective in speech and image recognition. At present, the risk management model based on deep learning has shown many advantages, such as powerful calculation, good adaptability, strong learning ability, and wide coverage, as well as high prediction accuracy and wide application. Although the application of deep learning algorithms in financial risk management has been matured, there are still many problems (Valeriane \& Wolfgang, 2020). Therefore, the study of financial credit risk management systems based on deep learning has important research value for promoting the development of the financial industry.

This paper aims to solve the application problems of deep learning in financial risk management. Given the insufficient credit scoring of the financial market, the background is introduced for the financial market risk management system, and some common deep learning algorithms are analyzed. Thereupon, the deep learning Bidirectional Recurrent Neural Network (BRNN) algorithm is introduced and used to implement the proposed model. In a BRNN, the output layer can get information both from past (backward) and future (forward) states simultaneously, thereby well-supplementing the absence of a feedforward neural network. Afterward, the BRNN algorithm is optimized by the bionic Particle Swarm Optimization (PSO) algorithm with simple operation and fast convergence speed to overcome the shortcomings, such as complex structure, slow convergence speed, and incomplete path analysis. Further, the AdaBound optimization algorithm is used to optimize the proposed BRNN model to avoid an extreme learning rate. Finally, the integrated financial market risk management system based on deep learning and the bionic algorithm is constructed, and the parameters and performance of the system are analyzed. The aim is to build a financial credit risk management system with higher accuracy than the latest research model. A case analysis further proves the effectiveness of the proposed model. Innovatively, the bionic algorithm is introduced into the risk management system. By combining the features of the bionic algorithm and deep learning algorithm, a model with faster convergence speed is implemented to process financial data efficiently. The research results are of great significance to reduce financial risks and improve the efficiency of financial market management. The proposal provides a reference for the combination of deep learning technology and bionic algorithm to address financial problems. This paper combines deep learning technology and the bionic algorithm to make up for their shortcomings in the field of financial market risk management and improve the convergence speed of the financial market risk management model. By comparing the performance in and between the single and integrated deep learning models, this paper deduces that integrated learning can improve classification accuracy and model generalization. Then, by analyzing the optimization results of different optimization algorithms under different data sets, it concludes that integrated learning can improve classification accuracy and model performance. Finally, it is proved that the accuracy of the financial credit risk management system based on the proposed integrated deep learning model is improved compared with other risk management systems in the latest research. 


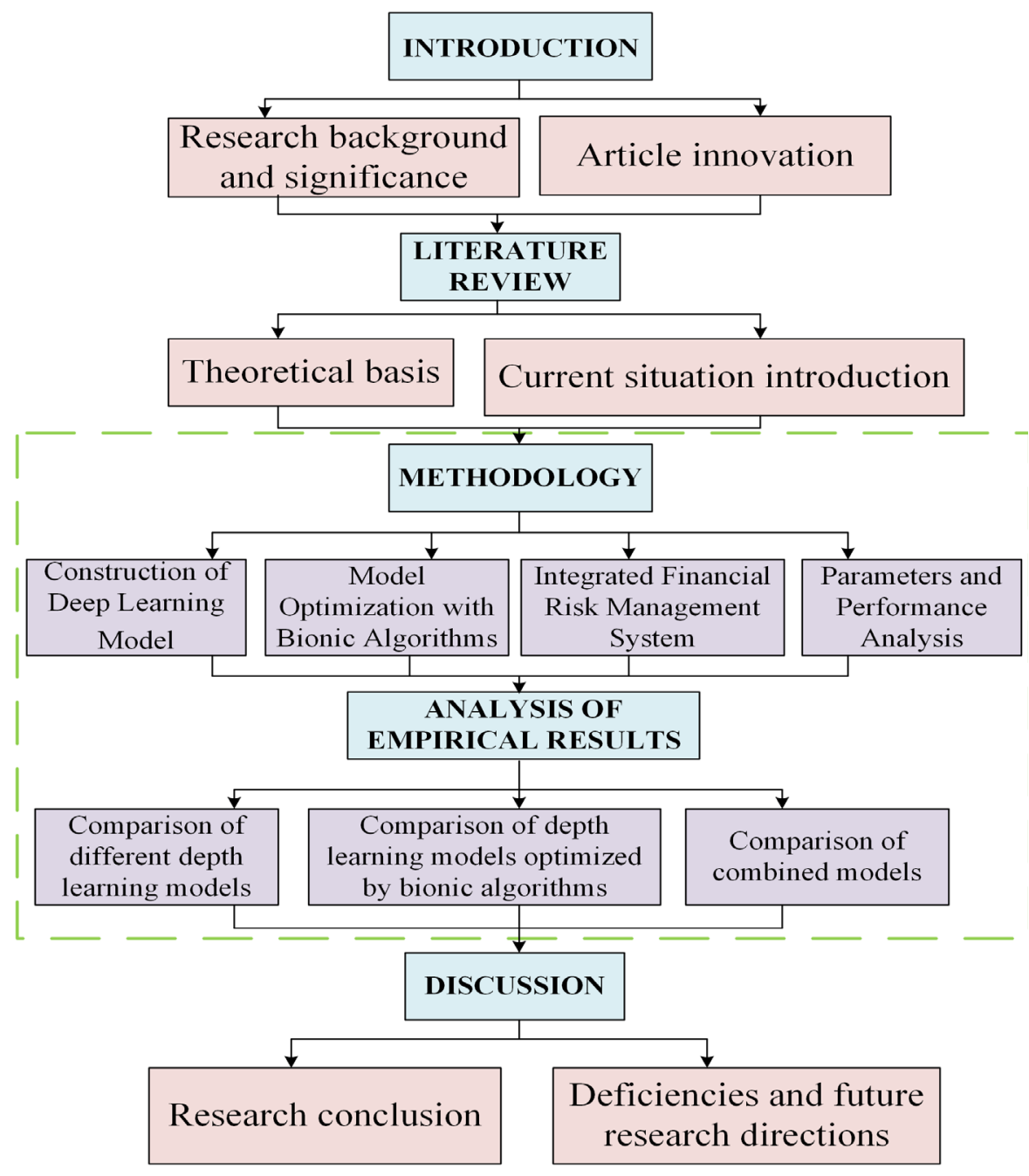

\section{LITERATURE REVIEW}

As presented in Figure 1, the core part of this paper is the model implementation and the results of empirical analysis. The main structure includes the introduction, research status, model implementation, empirical analysis results, and conclusions. The research status and model construction provide a theoretical basis for the results and conclusions, and the empirical analysis results provide data support for the conclusions. The second part is the theoretical support of this paper, which mainly introduces the research status of financial market risk management system, the application status of deep learning in the financial industry, and the current situation of financial market risk management based on deep learning and bionic algorithm.

\section{Financial Market Risk Management System}

Viktoriya et al. (2019) argued that financial risk management was an act of managing the potential risks of the financial market and protecting the economic value of enterprises through financial instruments (Viktoriya et al., 2019). Qiu (2021) constructed the enterprise risk management system through the 
VaR method by analyzing the data of different enterprises from 2012 to 2016 . The results showed that enterprise risk management was positively correlated with financial performance (Qiu, 2021). Tiwari et al. (2021) found that new financial instruments (deep learning, big data processing, and VAR model) could reduce the risk level but might also have some negative effects on the activities of financial market participants (Tiwari et al., 2021). Cox \& Lowrie (2021) improved the accuracy of financial risk management through Neural Network (NN) measurement and analysis model and provided a theoretical basis for financial risk management (Cox \& Lowrie, 2021). Kassi et al. (2019) analyzed financial risks based on return on assets, return on equity, and profit margin and found that different market risk management methods had a significant negative impact on the company's financial performance, while return on liabilities and stock turnover damaged the performance of nonfinancial companies (Kassi et al., 2019). Licia (2021) proposed an Internet Supply Chain Financing (SCF) credit risk management system based on data science. The model had a good piecewise fit for financial risk management and Supply Chain Management (SCM) under the Internet financial mode and high data evaluation accuracy (Licia, 2021). Yang et al. (2020) put forward an econometric analysis model of the financial market based on high-frequency data, which introduced two dimensions: government supervision and market free supervision. Research showed that the proposed method had good processing efficiency (Yang et al., 2020).

\section{Application of Deep Learning in Finance}

Deep learning has made many achievements in search technology, data mining, machine learning, Machine Translation (MT), Natural Language Processing (NLP), multimedia learning, speech recognition, and personalized recommendation technology, among others. Deep learning enables machines to imitate human activities. For example, Yang et al. (2021) found that deep learning could empower machines with vision, hearing, and thinking to solve complex pattern recognition problems to promote the development of Artificial Intelligence (AI) technology (Yang et al., 2021). The application of deep learning in the financial field has been matured. For example, Guo et al. (2019) built the stock selection model using the Convolutional Neural Network (CNN) for factor extraction, in which RNN was used for stock price prediction, and Long Short-Term Memory (LSTM) and RNN were combined for price regression or market trend classification (Guo et al., 2019). The deep learning algorithm can modify the model parameters autonomously, thus shortening the training time, optimizing the overall performance of the network, and improving the network generalization ability. Qian \& Olsen (2020) implemented the Ensem model by integrating the nonlinear deep learning for time series prediction based on NN, Support Vector Regression (SVR) machine, and extreme value optimization algorithm (Qian \& Olsen, 2020). Sha et al. (2020) proposed an innovative method and constructed a financial market risk management system based on AI. The financial portfolio of the system had high application value and was suitable for the existing financial market risk management (Sha et al., 2020). Bachute \& Subhedar (2021) constructed a new biological heuristic meta start method by combining the metaheuristic method with the deep learning model to realize the Spatio-temporal prediction of financial products (Bachute \& Subhedar, 2021). At present, the typical application of deep learning in the field of financial risk is the loss-sequence based deep learning model, which adapts the VaR algorithm and has higher accuracy than the traditional algorithms, and the performance has been verified through the bank risk monitoring and management system based on this model. Still, the traditional modeling methods for financial risk management have difficulty in mining complex data features or accurately reflecting such features as the financial cycle and easily ignores factors like economic development, actors' expectations, policy changes, and psychological changes, thereby lowering financial risks predictability. Besides, the traditional modeling methods rely heavily on researchers' subjective design and lack objectivity. For example, traditional linear methods often require strong linear assumptions, while traditional machine learning methods cannot efficiently process noise signals. These problems restrict the accurate analysis and prediction of financial market data (Liu et al., 2021). 


\section{Summary of Previous Research}

To sum up, the existing scholars mostly adopt statistical and simple machine learning methods in financial market risk management. Although machine learning methods can solve simple and unconstrained problems, according to the research of Akber et al. (2021) and Flammer and Ioannou (2021), it is not suitable to address complex dimensional data or massive amounts of data (Akber et al., 2021; Flammer \& Ioannou, 2021). By contrast, the deep learning method can learn independently, and simplify the classification and prediction problems through dimension reduction. Yet, the research of Sun \& $\mathrm{Li}$ (2021) showed that in the actual application in financial market risk management, deep learning still faced some problems that hindered its further popularization, such as insufficient credit scoring, incomplete path analysis, extreme learning rate (Sun \& Li, 2021). Given these deficiencies, this paper introduces corresponding optimization algorithms and verifies the effectiveness of the proposed algorithm through data analysis, including the BRNN for insufficient credit scoring and PSO algorithm to optimize the path analysis, as well as the AdaBound algorithm to improve the learning efficiency by reducing the learning rate. Finally, the integrated financial market risk management system based on deep learning and the bionic algorithm is constructed. The effectiveness of the constructed model is further proved through case analysis.

\section{METHODOLOGY}

The methodology section is the core of this paper. This section will build a deep learning model and optimize the bionic algorithm model. Thereupon, an integrated risk management model based on deep learning and the bionic algorithm is implemented. Finally, the parameters and performance of the model are analyzed with an example.

\section{Construction of Deep Learning Model}

Deep learning shows high prediction accuracy in practice, through which the underlying data patterns can be obtained. Thus, deep learning can better handle large and high-dimensional datasets. Meanwhile, the deep learning model is easier to update given new data, and the hidden layers of the deep learning model significantly reduce the feature engineering. Hence, deep learning is the latest technology suitable for computer vision, MT, emotion analysis, and speech recognition (Chen et al., 2020). Deep learning mainly features strong learning ability and significant processing effect, which enhance its popularity. Currently, most existing credit scoring models are implemented with shallow structures, so deep learning is innovatively introduced into the credit scoring model, and the RNN model and BRNN are used for credit scoring. RNN and BRNN can share parameters, memorize data information through the network, learn sequential nonlinear features efficiently, and are widely used in NLP and computer vision, speech recognition, MT, and text classification (Hassib et al., 2018). Under this inspiration, the RNN and BRNN are applied to the credit scoring model, in which customer credit behaviors are taken as a sequence, and each credit feature is taken as a vector. Since the deep learning model has enough hidden layers and strong feature learning ability, the RNN and BRNN can be used in memory networks, and network loss can be reduced by summing up the loss functions at all moments, thus ensuring a high credit scoring accuracy. Additionally, BRNN is the superposition of two single-directional RNNs, avoiding the limitation of a regular RNN. BRNN can excavate the potential relationship between each credit data more deeply by associating forward and backward states and improve the prediction accuracy based on the customer's overall credit data. Figure 2 shows the network structure of a single-layer BRNN.

The output of the forward state is not connected to the input of the backward state. At time $t$, the hidden layer $h_{t 1}$ of the forward RNN and the hidden layer $h_{t 2}$ of the backward RNN can be expressed as in Eqs. (1) and (2), respectively. 
Figure 2. Network structure of a single-layer BRNN

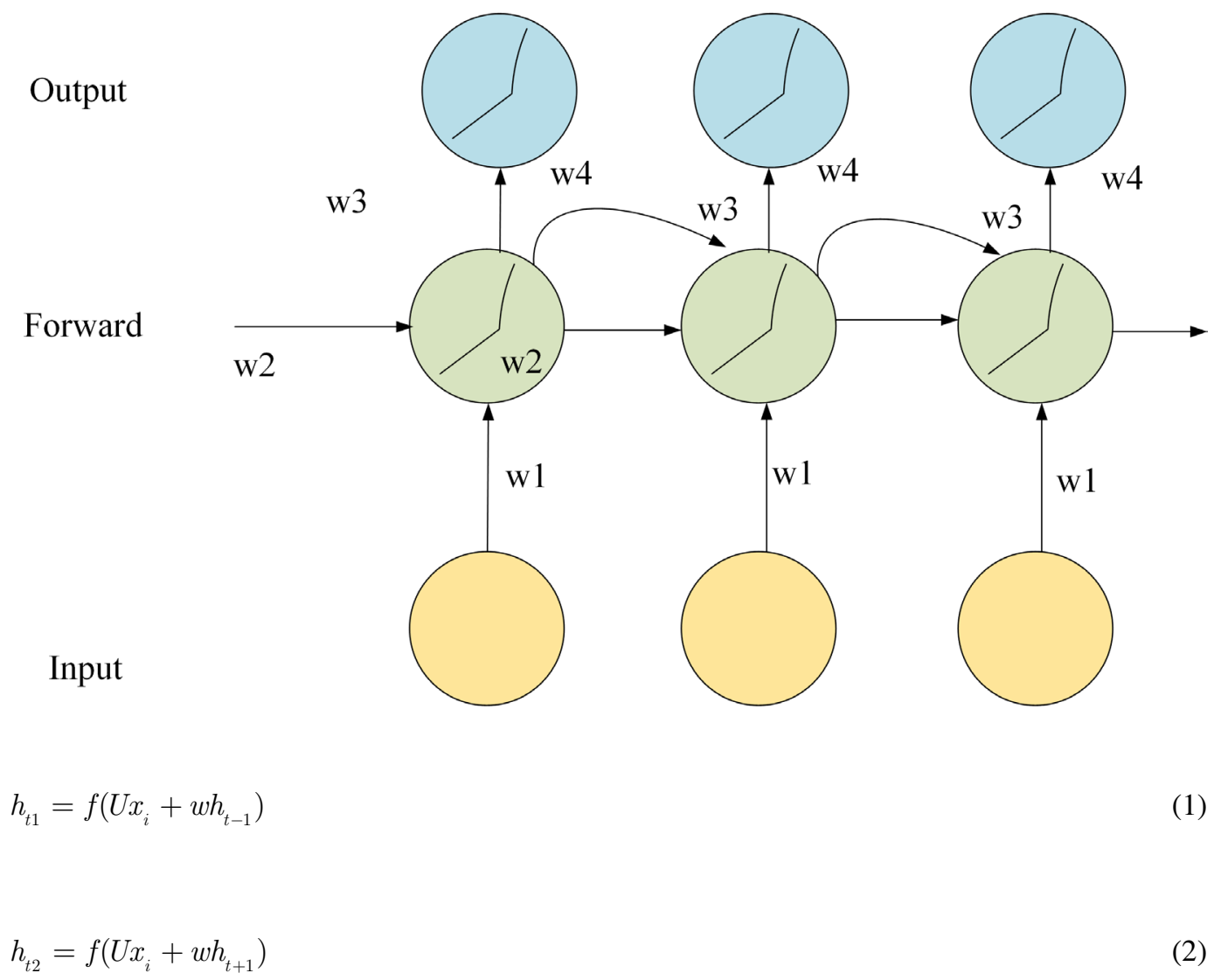

In (1) and (2), $x_{i}$ denotes the input value, $h_{t-1}$ stands for the previous hidden layer, $h_{t+1}$ represents the next hidden layer, and $\mathrm{w}$ is the weight size. The prediction output $y_{t}$ of the single-layer BRNN can be expressed as in Eq. (3).

$y_{t}=\operatorname{soft} \max \left(\mathrm{g}\left(\mathrm{V} x_{i}+V h_{t+1}\right)\right)$

Deep BRNN refers to a BRNN network with more than two hidden layers. A multi-layer network is used for every input, so the network has stronger expression ability and learning ability but also higher complexity (Zhou et al., 2021). At time $t$, the hidden layer can be expressed as in Eq. (4).

$$
h_{t}=f\left(U h_{t}+\omega h_{t-1}\right)
$$

The $i$ th hidden layer at time $t$ can be expressed as in Eq. (5).

$$
h_{t}=f\left(U h_{t}+\omega h_{t+1}\right)
$$

If the deep BRNN has L hidden layers, then the final prediction output can be expressed in Eq. (6). 
$y_{t}=\operatorname{soft} \max \left(\mathrm{g}\left(\mathrm{V} h_{t}+V h_{t+1}\right)\right)$

The BRNN is trained through a time backpropagation algorithm, which is essentially a Gradient Descent (GD) method. It iteratively searches for better points along the negative gradient direction of parameters to be optimized until the algorithm is converged. The specific structure is shown in Figure 3.

Figure 3. Network structure of double-layer BRNN

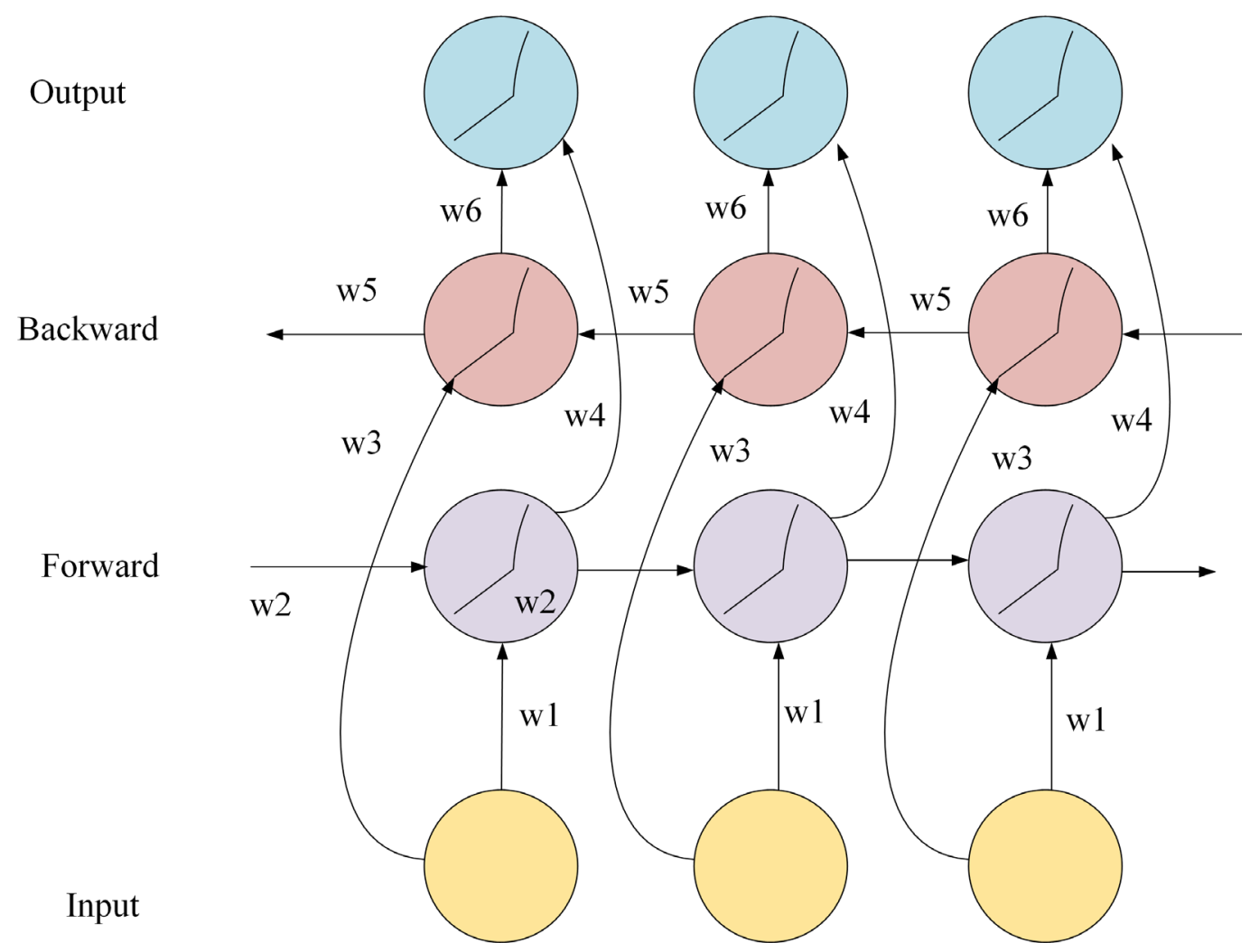

\section{Model Optimization with Bionic Algorithms}

Subsequently, the AdaBound bionic algorithm is introduced to optimize the proposed BRNN model in path analysis (Tang et al., 2021). Compared with the Stochastic Gradient Descent (SGD) algorithm, the adaptive optimization method has weak generalization, and an extreme learning rate will lead to poor performance (Shi et al., 2020). Therefore, there is a need to construct a more suitable algorithm that can combine the advantages of adaptive optimization and the SGD algorithms with fast initialization and convergence, as well as good generalization. The AdaBound bionic algorithm is similar to the adaptive optimization method (Adam Optimization algorithm) in its early training stage; in the learning rate update phase, the learning rate is first trimmed and controlled within a dynamic change; as the time step increases, the upper and lower boundaries get closer, and, finally, converge to a fixed value; at this time, the learning rate is also fixed, and the AdaBound algorithm is transformed into an SGD algorithm (Liang et al. 2021). AdaBound algorithm avoids extreme 
learning rates through trimming and boundary control. In the early stage of training, it is similar to the Adam algorithm and shows a very fast initialization. With the convergence of the learning rate, the AdaBound algorithm gradually and smoothly turns into an SGD algorithm in the late stage of training and shows good generalization. The specific process is shown in Figure 4.

Figure 4 indicates that the model optimization process of the bionic algorithm consists of several parts, including index input, the decay rate calculation, the parameter initialization, and the termination condition. If the termination condition is satisfied, the parameters are directly updated and output; otherwise, a small number of samples are selected to update the matrix. Finally, the learning rate of the algorithm is trimmed to update the parameters and output. The core step of the process is the trimming control when the learning rate is updated. The AdaBound algorithm used here maintains a stable learning rate range by trimming and boundary control.

\section{Integrated Financial Risk Management System}

The parallel integration method is adopted for the integrated financial risk management system, which combines the results of different base classifiers. There is no strong dependence among the base classifiers, and each base classifier learns parallelly to generate an independent model. The final result is obtained through the combined scores of each base classifier. The integrated financial risk management system is constructed following five steps: data preprocessing, construction of a single classification model, evaluation of a single classification model, construction of an integrated classification model, and evaluation of the integrated classification model. Evaluation indexes are used to measure the performance of the classifier, as well as the performance of the single classification model, and the first three single classification models with the highest scores are selected to implement the integrated classification model. The score calculation of a single classification model is shown in Figure 4. The calculation of the Total-score reads:

$$
\text { Total-score }=\sum_{i=1}^{k} \omega_{i} X_{i}
$$

In (7), $\mathrm{X}_{\mathrm{i}}$ represents the evaluation index of the ith classifier, and $\mathrm{w}_{\mathrm{i}}$ stands for the corresponding weight.

Figure 5 illustrates the prediction results of the eXtreme Gradient Boosting (XGBoost), Logistic Regression (LR) classifier, Dense Trajectory (DT), K-Nearest Neighbor (KNN) classification, Support Vector Machine (SVM), Linear Discriminant Analysis (LDA), Random Forest (RF), RNN, and BRNN algorithms. The algorithm performance is evaluated from the Area Under Curve (AUC), Adaptive Cruise Control (ACC), and F-score. Finally, the comprehensive performance of the single classification model and the integrated classification model is presented.

According to the characteristics of the dataset and practical experience, the weight of each classifier evaluation index is set as Eq. (8).

$$
\omega_{A U C}: \omega_{A C C}: \omega_{F-\text { score }}: \omega_{T 1}: \omega_{T 2}=0.5: 0.3: 0.2:-0.3:-0.2
$$

In (8), $\mathrm{w}_{\mathrm{n}}$ represents the weight of different indexes. After evaluation, the single classification models are combined into seven integrated classification models, and then the performance of the seven integrated classification models is compared following the evaluation indexes, as shown in Figure 6. The experimental dataset is divided into the test set and the training set with a ratio of 2:8. The results of 10 experiments are averaged to obtain the final experimental result. 
Figure 4. Model optimization process using bionic algorithms

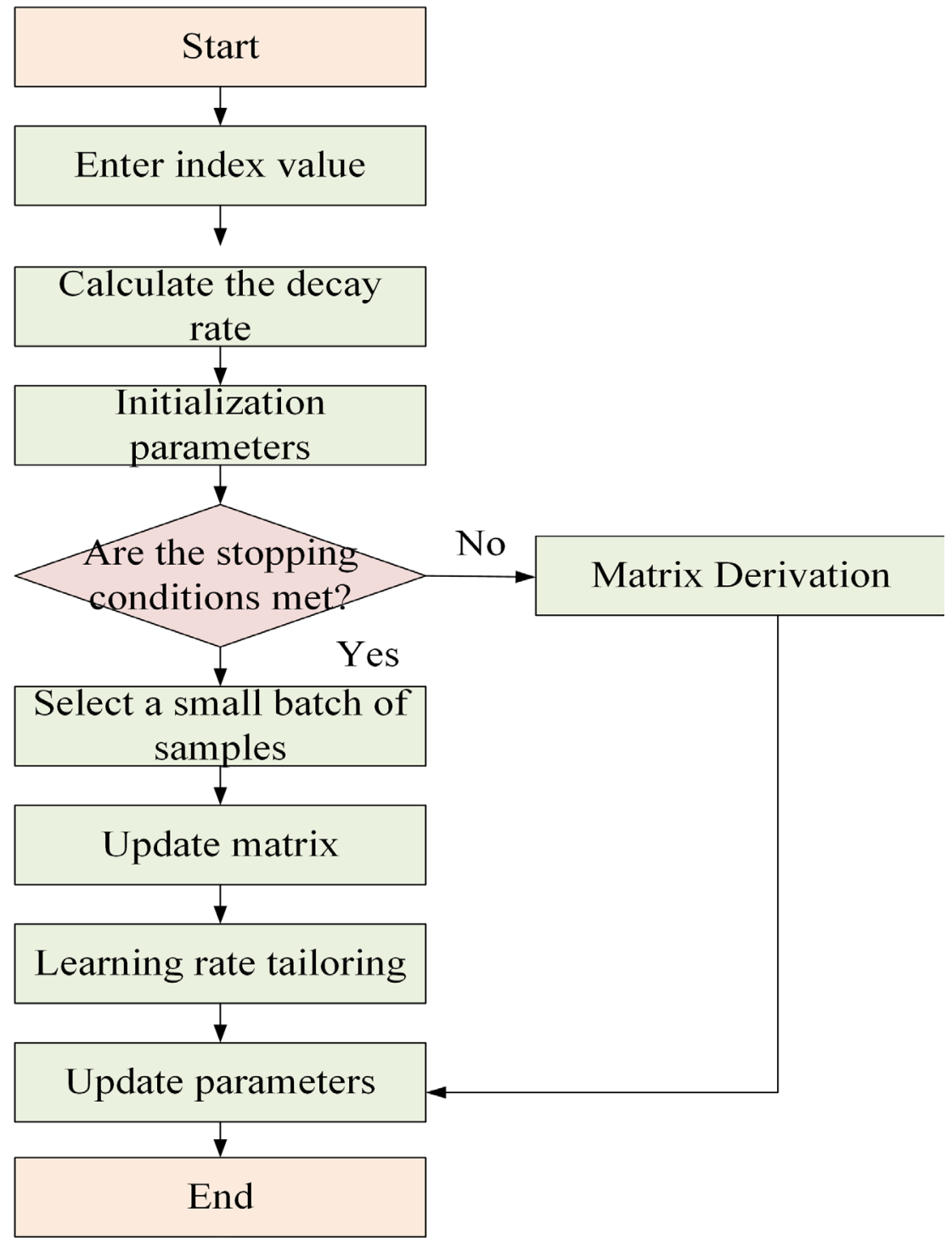

The algorithms involved in Figure 6 are consistent with those in Figure 5. The integrated financial risk management system includes the BRNN, as well as the training set processing, parameter normalization, missing value processing, and dataset initialization. 
Figure 5. Credit scoring flowchart of the single classification model

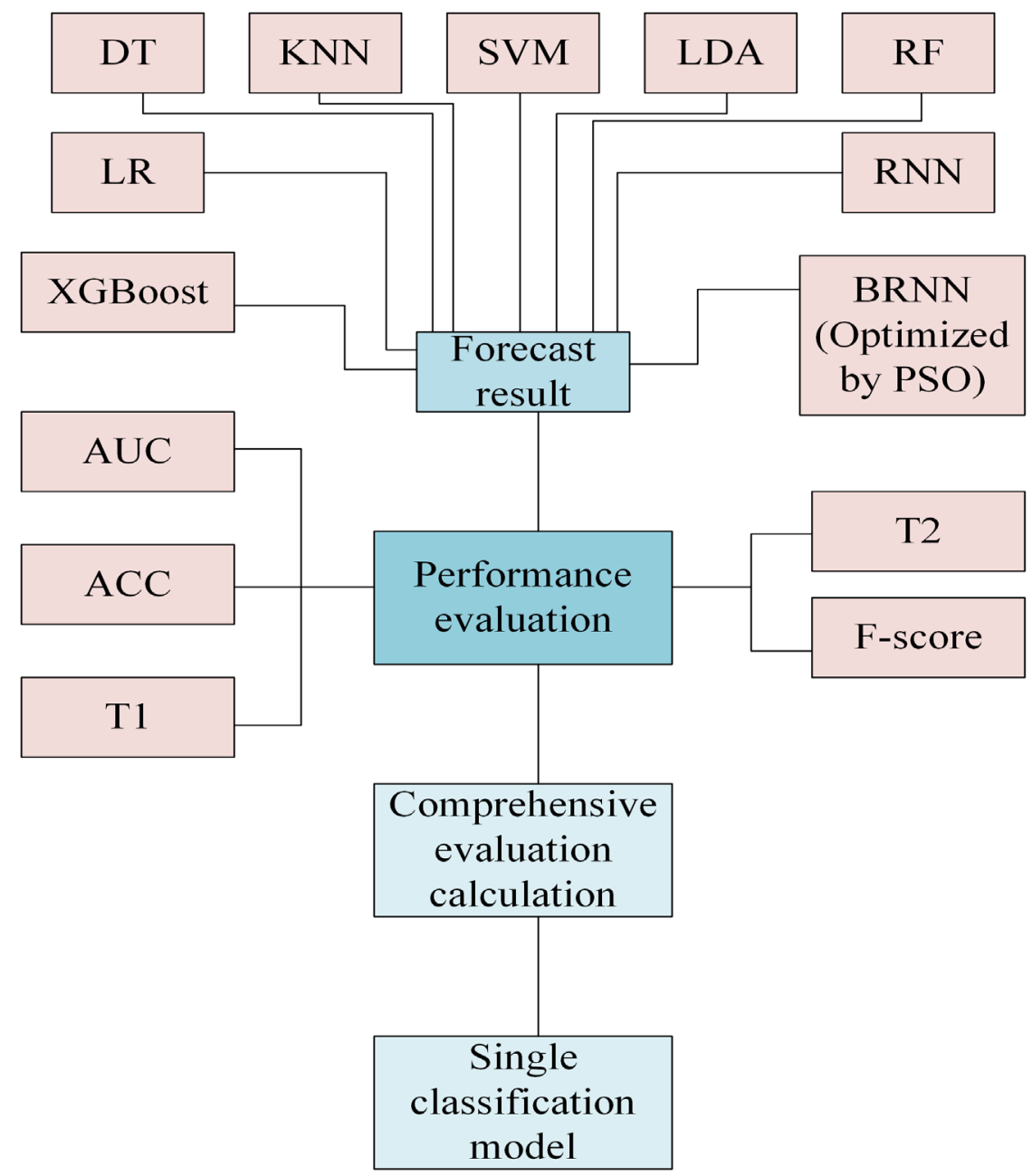

\section{Parameters and Performance Analysis}

(1) Data sources. The experimental financial dataset is taken from the University of California Irvine (UCI) machine learning database of Australia, Germany, and Japan (Crivello et al., 2021). The specific sample information is shown in Table 1. First, the sample data are preprocessed, the missing feature is assigned with 0 , and dummy variables are defined to replace the attributes of classification categories with 1, 2, and 3 respectively. Finally, the data are normalized.

(2) Parameter setting. Different datasets are set with different parameters, as shown in Table 2. Gbtree is chosen as the classifier, the learning rate is set to 0.03 , the node weight in the training set is 0.1 , the maximum depth is 3 , the proportion of random sampling is 0.7 , and the proportion of feature selection is 0.8 . The number of $\mathrm{CNN}$ neurons is set differently for different datasets, Tanh 
is used for the hidden layer of the activation function, and SoftMax is used as the output layer. All comparable models use the same dataset as the proposed integrated deep learning model.

(3) Performance analysis. Model performance evaluation can be transformed into a binary classification problem using the confusion matrix (QuezadaTéllez et al., 2021). Table 3 shows the confusion matrix, TP is the true positive proportion, namely, the proportion of accurately predicted positive samples in the real samples, and TN is the true negative proportion, namely, the proportion of accurately predicted negative samples in the real samples. FP is the false-positive proportion, namely, the proportion of positively predicted negative samples in the real samples, and FN is the false negative proportion or the proportion of negatively predicted positive samples in the real samples.

Figure 6. Integrated financial risk management system

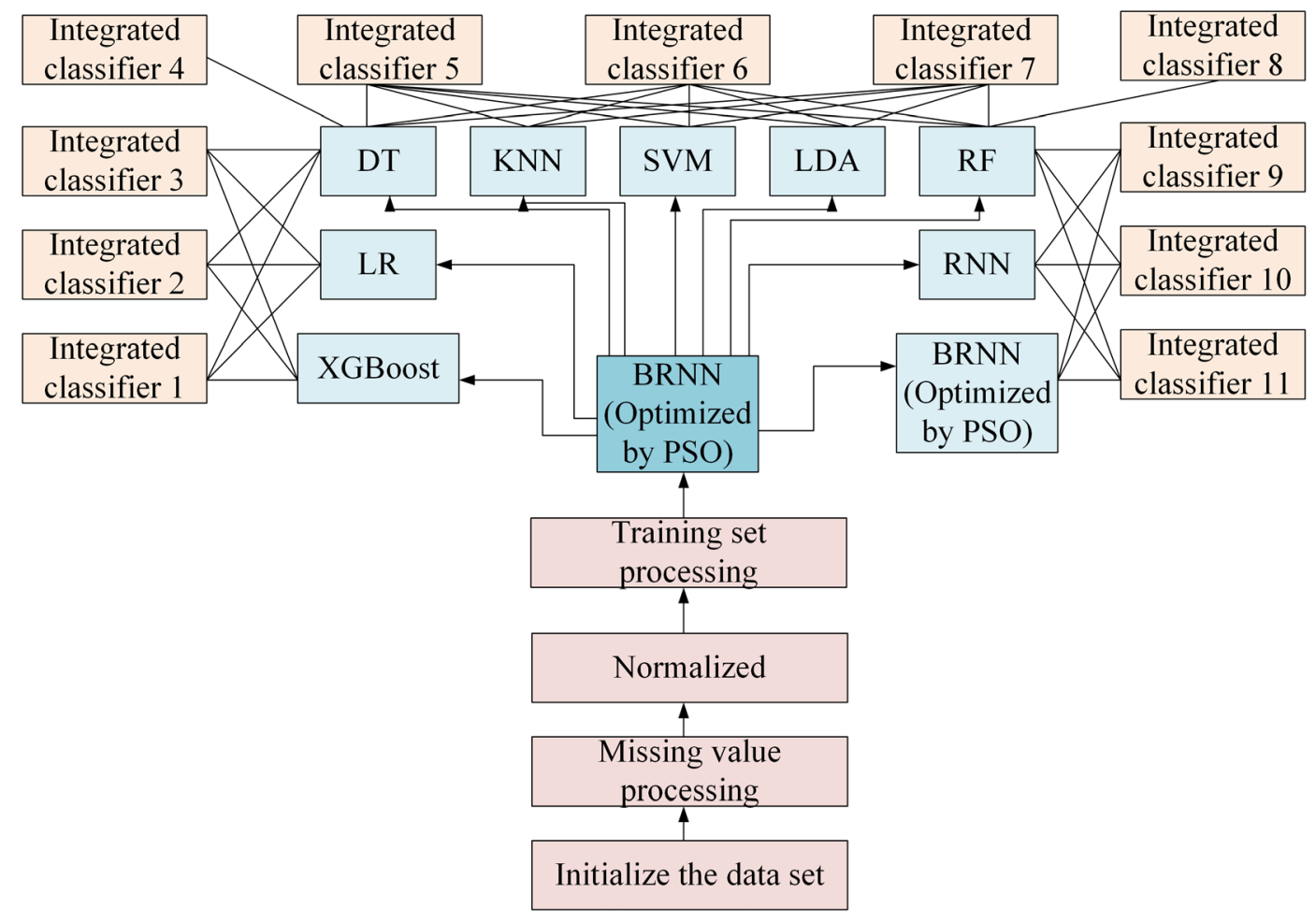

Table 1. Sample dataset information

\begin{tabular}{|l|l|l|l|l|}
\hline \multicolumn{1}{|c|}{ Dataset } & \multicolumn{1}{|c|}{ Samples number } & Features number & \multicolumn{1}{|c|}{$\begin{array}{c}\text { Positive sample } \\
\text { number }\end{array}$} & $\begin{array}{c}\text { Negative sample } \\
\text { number }\end{array}$ \\
\hline Australia & 720 & 12 & 318 & 394 \\
\hline Germany & 1103 & 18 & 702 & 324 \\
\hline Japan & 685 & 16 & 313 & 375 \\
\hline
\end{tabular}


Table 2. Parameter settings

\begin{tabular}{|l|l|l|l|}
\hline \multicolumn{1}{|c|}{ Parameters } & \multicolumn{1}{c|}{ Setting } & \multicolumn{1}{c|}{ Parameters } & \multicolumn{1}{c|}{ Setting } \\
\hline Classifier & Gbtree & $\begin{array}{l}\text { Number of hidden layer } \\
\text { neurons }\end{array}$ & $8 / 15 / 8$ \\
\hline $\begin{array}{l}\text { Classification learning } \\
\text { rate }\end{array}$ & 0.03 & Number of hidden layers & $3 / 4 / 3$ \\
\hline Node weight & 0.1 & Convolution LR & 0.001 \\
\hline Maximum depth & 3 & Batch size & $552 / 800 / 552$ \\
\hline Random sampling ratio & 0.7 & Iteration times & $3000 / 4000 / 3000$ \\
\hline Feature ratio & 0.8 & Neuron loss rate & 0.3 \\
\hline
\end{tabular}

Table 3. Confusion matrix

\begin{tabular}{|l|l|l|l|}
\hline \multirow{3}{*}{ Real situation } & & \multicolumn{2}{|c|}{ Prediction results } \\
\cline { 2 - 4 } & Positive samples & Positive samples & Negative samples \\
\cline { 2 - 4 } & Negative samples & True Positive Proportion (TP) & $\begin{array}{l}\text { False Negative Proportion } \\
(\mathrm{FN})\end{array}$ \\
\cline { 2 - 4 } & False Positive Proportion (FP) & $\begin{array}{l}\text { True Negative Proportion } \\
\text { (TN) }\end{array}$ \\
\hline
\end{tabular}

The main evaluation indexes: AUC is the area under Receiver Operating Characteristic (ROC) curve, and the higher its value, the better the classification effect. ACC is the proportion of correctly predicted samples in the total sample. Precision (Pre) represents the proportion of real positive samples in the predicted positive samples. Type I error (T1) is the proportion of real negative samples predicted as positive samples in all real negative samples. Type II error (T2) stands for the proportion of real positive samples predicted as negative samples in all real positive samples. F-score is a comprehensive index based on precision and recall: the closer F-score is to 1, the better the classification effect is. The specific calculation reads:

$$
A C C=\frac{T P+T N}{T P+T N+F P+F N}
$$

$\operatorname{Pr} e=\frac{T P}{T P+F P}$

$$
T 1=\frac{F P}{F P+T N}
$$




$$
T 2=\frac{F N}{T P+F N}
$$

$$
F \text {-score }=\frac{2 \times \text { pre } \times T P R}{p r e+T P R}
$$

The training samples are randomly selected. The software and hardware environments are configured with a Windows 10 64-bit operating system, a 16G RAM, the TensorFlow framework based on Python 3.6, and an Intel Core (TM) i5-3337U CPU with a frequency of $1.8 \mathrm{GHz}$.

\section{ANALYSIS OF EMPIRICAL RESULTS}

The results are the empirical research results of the model based on deep learning and bionic algorithm, including the performance comparison of different deep learning models under different data sets, the performance comparison of different integrated deep learning models, the optimization results of the bionic algorithm on different deep learning models, and the application effect of integrated deep learning model. This section provides data support for the conclusion (Shen et al., 2019).

\section{Performance Comparison of Deep Learning Models}

Figures $7 \mathrm{a}-7 \mathrm{c}$ show the performance indexes of different single model algorithms on the three datasets of Australia, Germany, and Japan. Apparently, the F-scores of LR, XGBoost, and BRNN are better in the Australian data set, and the XGBoost model shows the highest AUC of 0.9384; the LR model presents the highest F-score of 0.8557, and the BRNN model has the lowest T1 rate of 0.0965. In the German dataset, LR, XGBoost, and BRNN have better classification effects; the XGBoost shows the highest AUC of 0.8049 , as well as the highest accuracy of 0.7750 . For the Japanese dataset, LR, XGBoost, and BRNN perform better; the XGBoost presents the highest AUC of 0.9307.

In Figure 7, the $\mathrm{X}$-axis represents the algorithm category, the left $\mathrm{Y}$-axis indicates the specific calculation method category, and the right $\mathrm{Y}$-axis denotes the comprehensive performance parameter. Figures 8A-8C display the comprehensive performance of single model performance on different datasets. In the Australian dataset, XGBoost and BRNN have the highest comprehensive performance, reaching 0.83 , followed by $\mathrm{LR}$, with 0.82 . In the German data set, the BRNN shows the highest comprehensive performance (0.63), followed by the LR model (0.62-0.63), and the comprehensive performance of XGBoost is 0.62 , ranking third. In the Japanese dataset, the BRNN model presents the highest comprehensive performance, reaching 0.83, followed by the XGBoost model, and the third is the LR model with a comprehensive performance of 0.82 . Thus, the comprehensive performance of LR, XGBoost, and BRNN is better than that of other models on each dataset. Therefore, these three models are combined heterogeneously.

In Figure 8, the $\mathrm{X}$-axis indicates the algorithm type, and the $\mathrm{Y}$-axis represents the comprehensive performance parameter.

\section{Performance Comparison of Integrated Deep Learning Models}

In Figure 9, the X-axis represents the performance index of different integrated classification models on different datasets, and Y-axis denotes the types of integrated classification algorithms. Figures 8A-8C demonstrate the performance indexes of different integrated classification models on different datasets. For the Australian dataset, the BRNN + LR + XGBoost model has the highest AUC of 0.9574 , the highest accuracy of 0.8993 , the lowest $\mathrm{T} 1$ rate of 0.0842 , and the highest F-score of 0.8871 . 


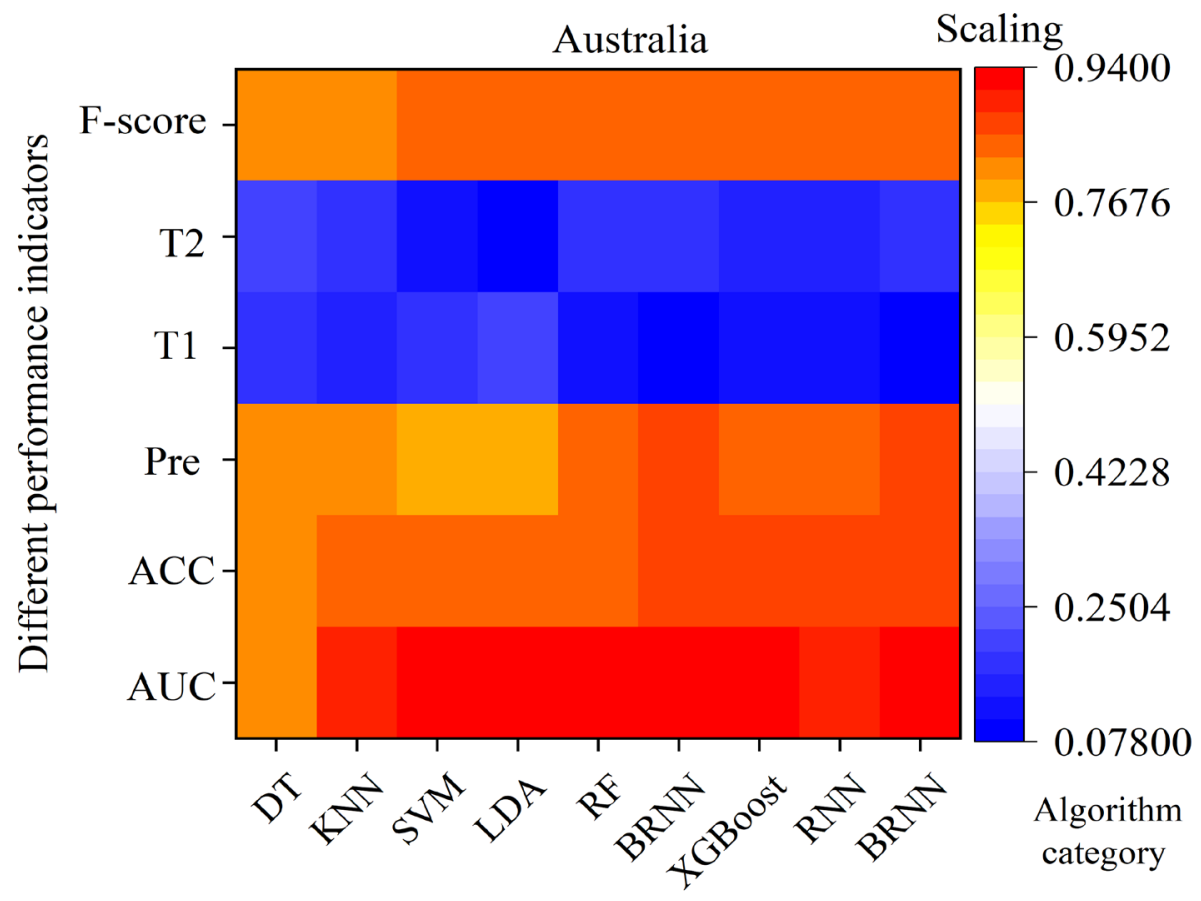

Figure 7b. Performance of single classification model on different datasets

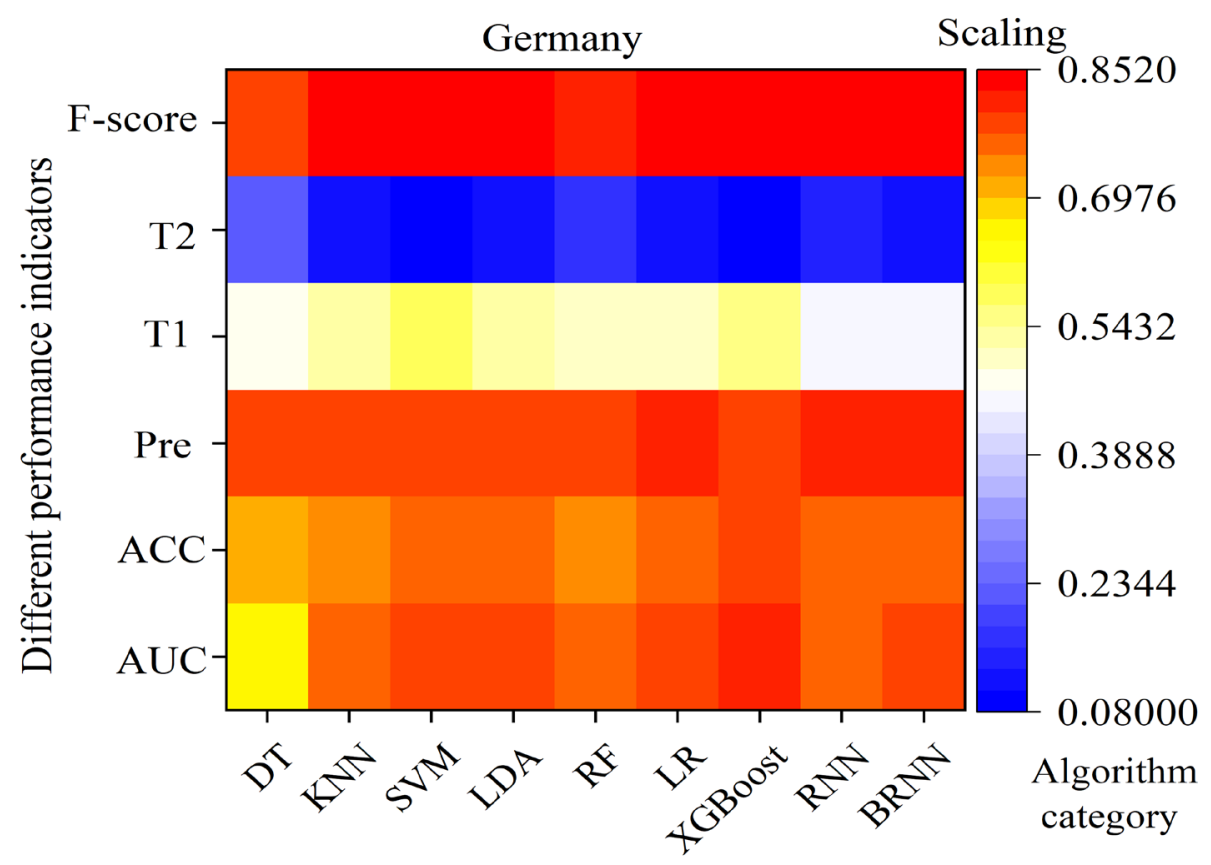


Figure 7c. Performance of single classification model on different datasets

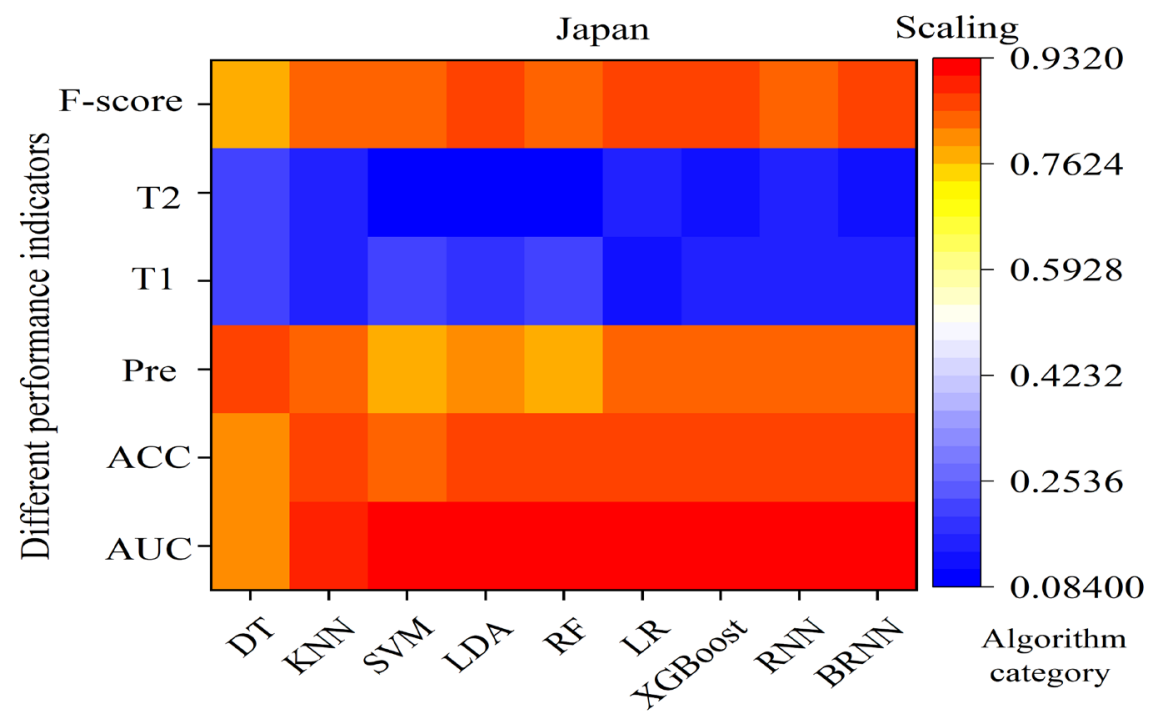

Figure 8a. Comprehensive performance of single classification model on different datasets

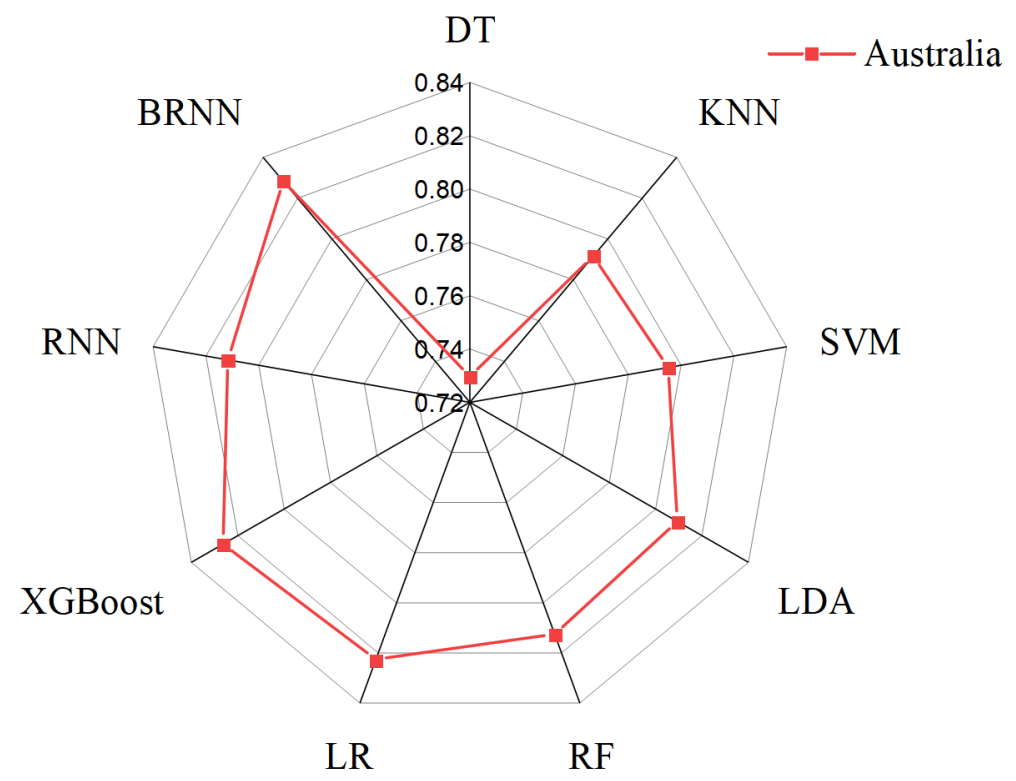


Figure 8b. Comprehensive performance of single classification model on different datasets

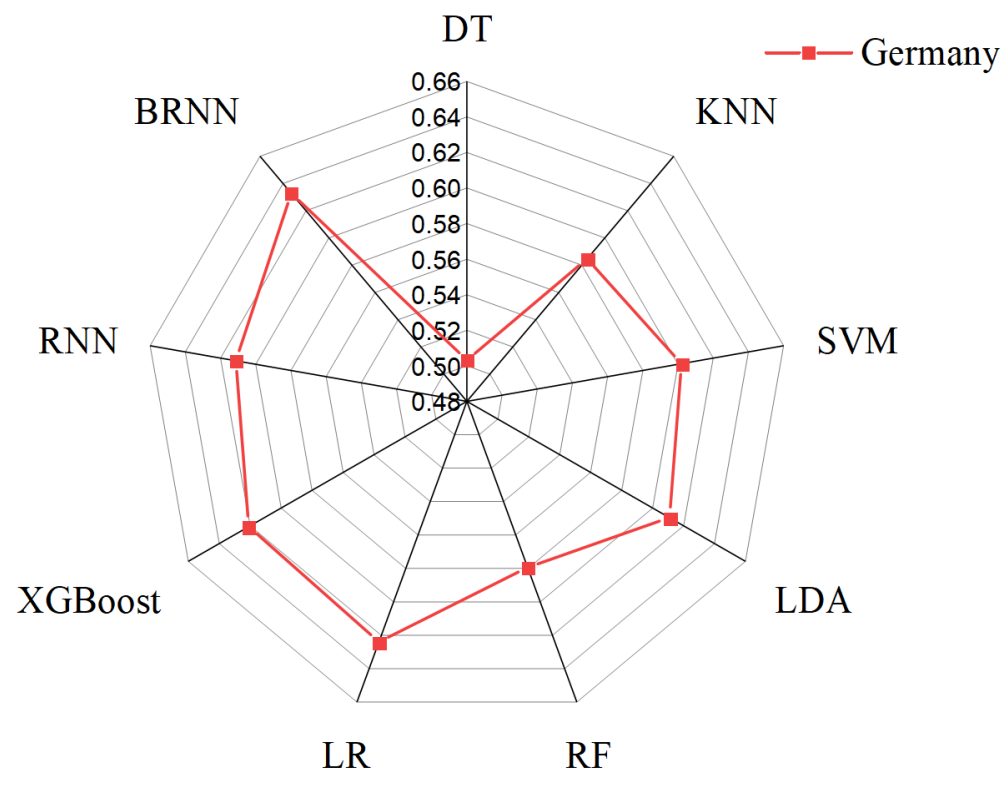

Figure 8c. Comprehensive performance of single classification model on different datasets

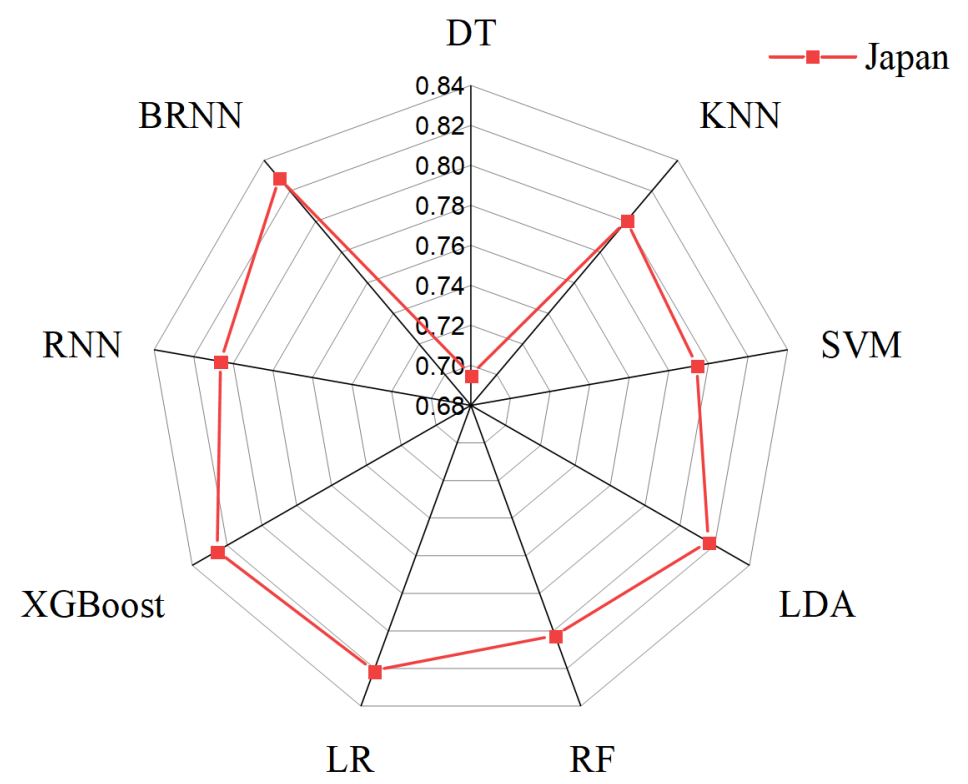


Figure 9a. Performance indexes of different integrated classification models on different datasets

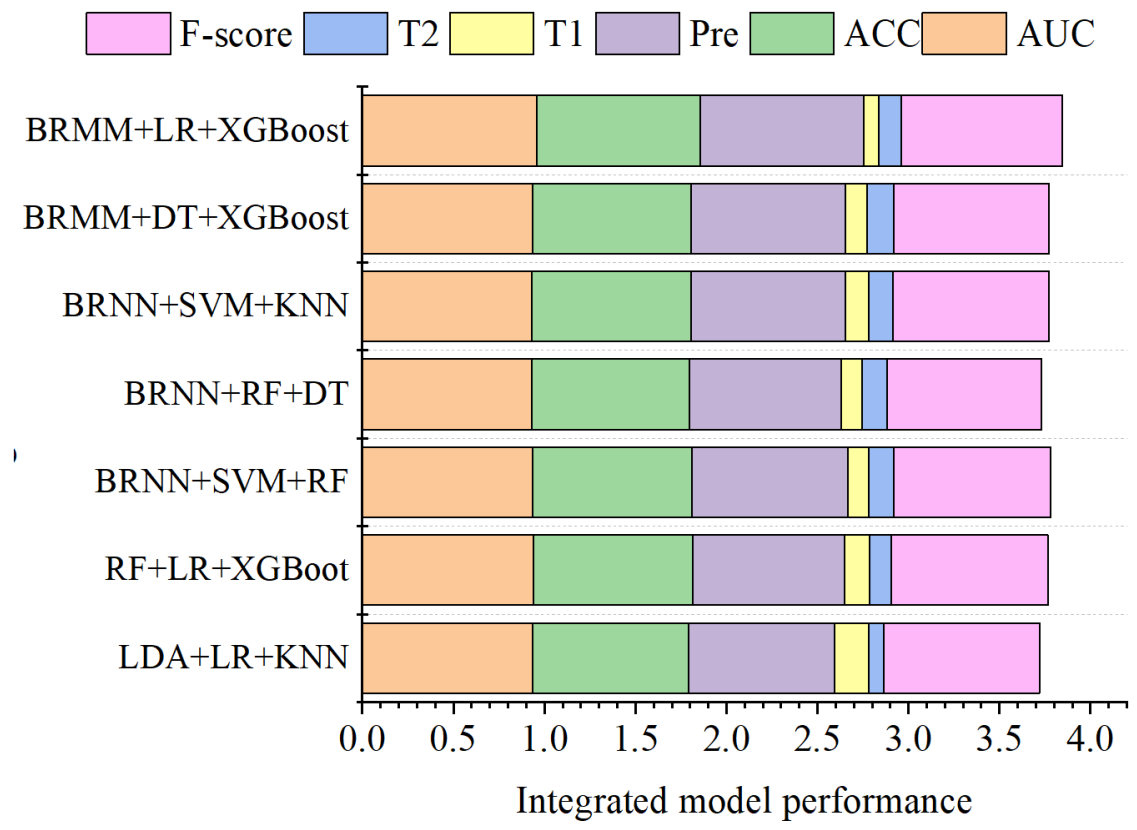

Figure 9b. Performance indexes of different integrated classification models on different datasets

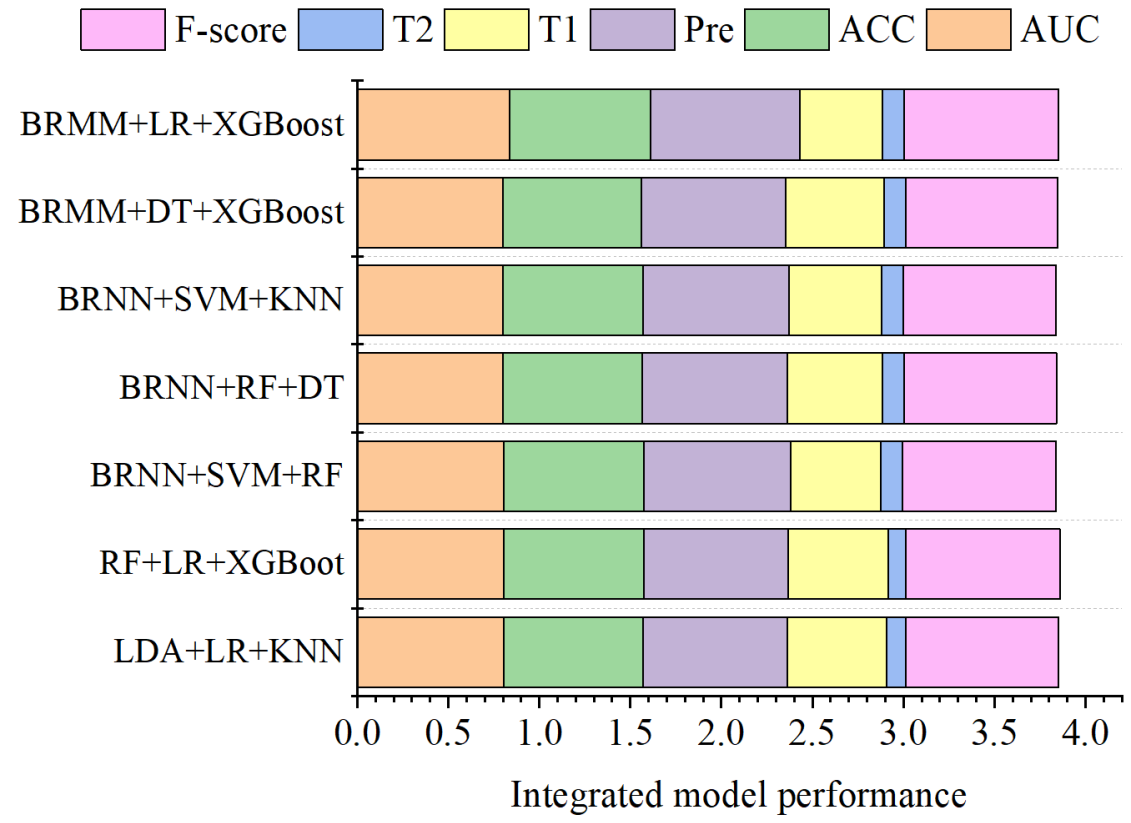


Figure 9c. Performance indexes of different integrated classification models on different datasets

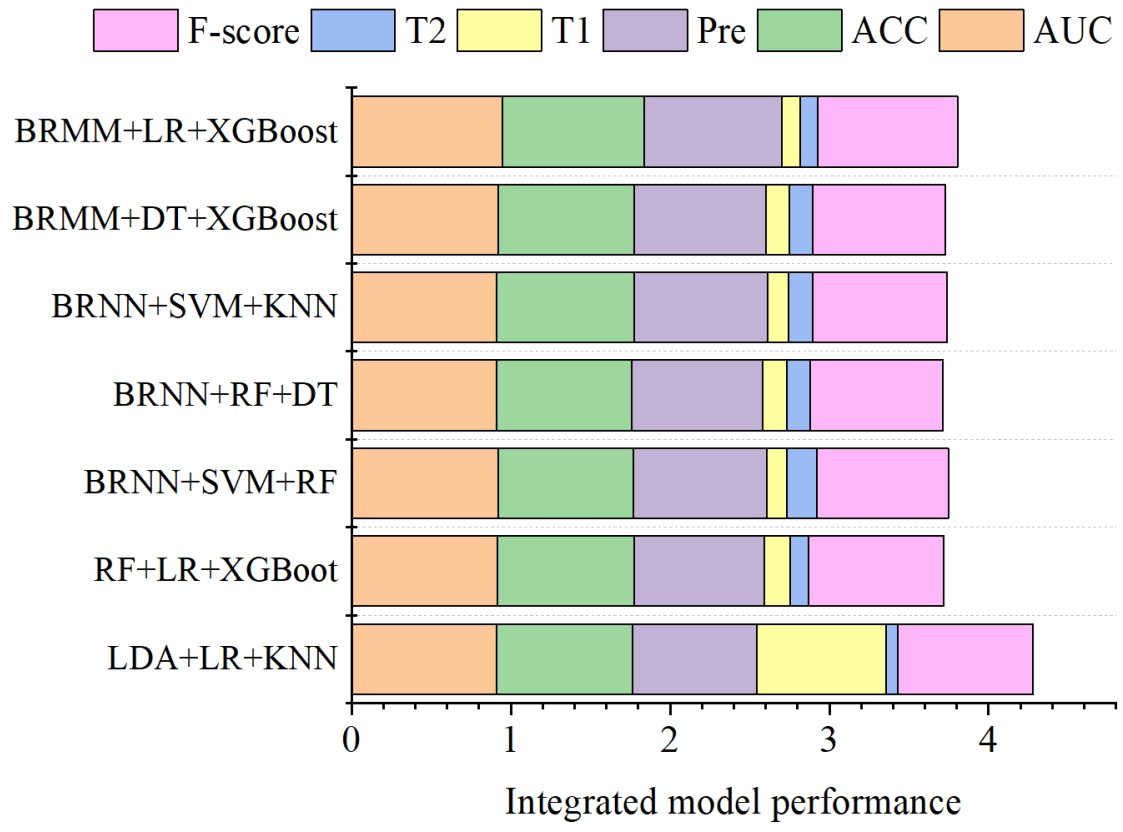

Figure 10a. BRNN model optimization results of different algorithms with different datasets

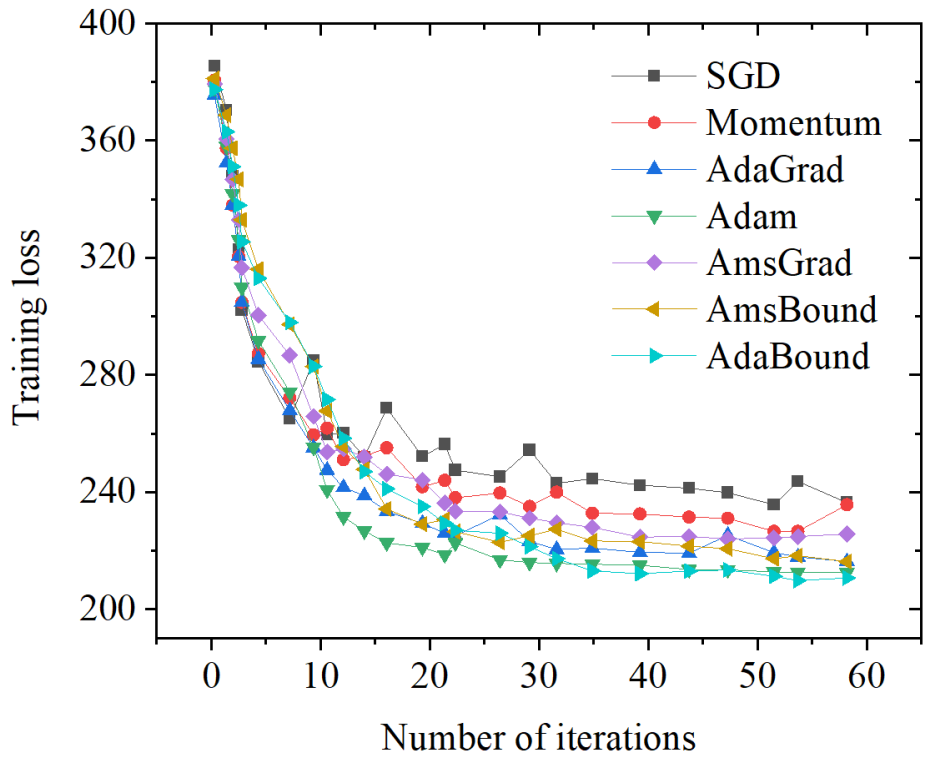


Figure 10b. BRNN model optimization results of different algorithms with different datasets

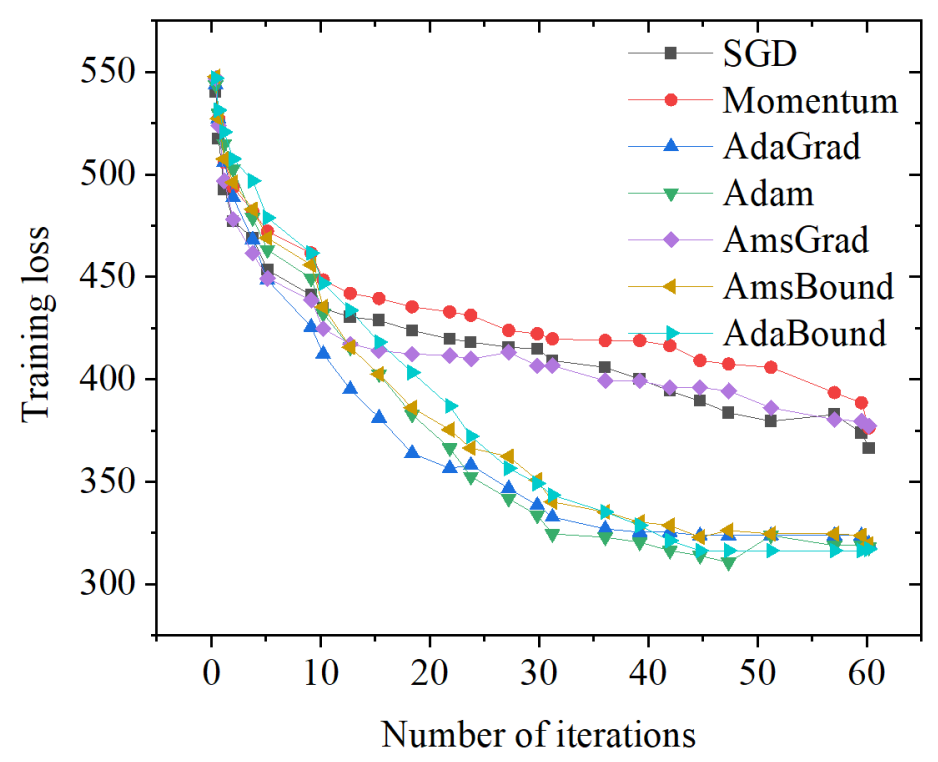

Figure 10c. BRNN model optimization results of different algorithms with different datasets

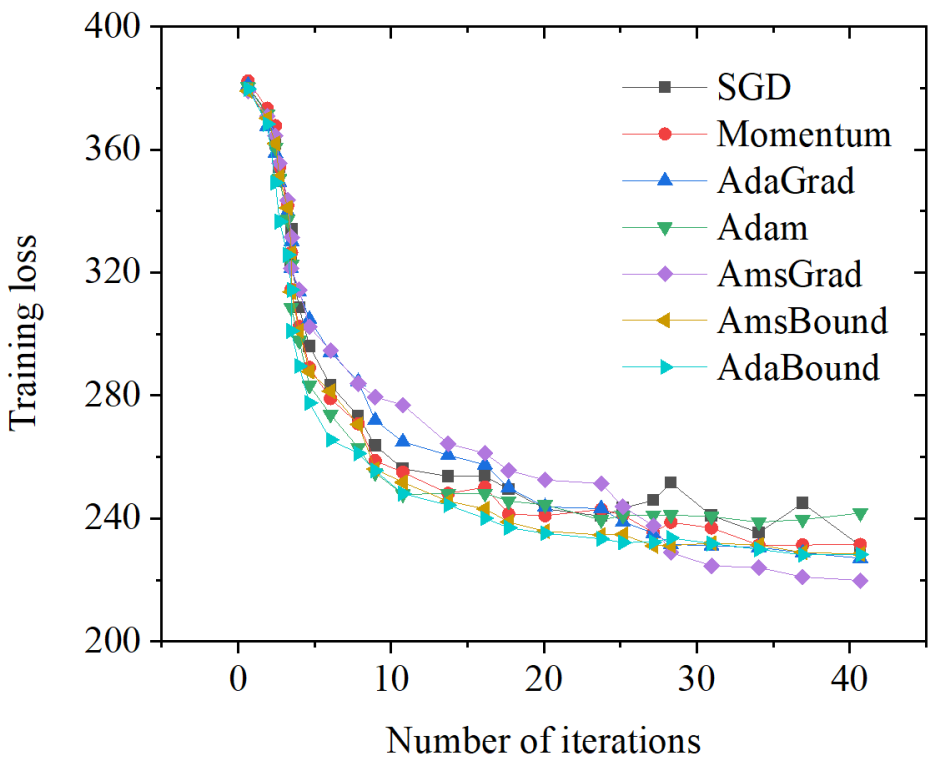


For the German dataset, the BRNN + LR + XGBoost model shows the best classification effect and performance; the highest AUC is 0.8374 , the highest accuracy is 0.7750 , the highest precision is 0.8190 , the lowest $\mathrm{T} 1$ rate is 0.4553 , and the highest F-score is 0.8510 . For the Japanese dataset, the BRNN + LR + XGBoost model has the best performance, with an AUC of 0.9491, an accuracy of 0.8876 , and an F-score of 0.8773 . The results prove that integrated learning can improve classification accuracy and model generalization.

\section{Optimization Results of the Bionic Algorithms on Different Deep Learning Models}

Figures 10a-10c illustrate the optimization results of different optimization algorithms with different datasets. For the Australian dataset, the Adam algorithm has the fastest initial progress, the fastest convergence speed, and the smallest training loss, SGD algorithm has the slowest convergence speed and the largest training loss, and AdaBound algorithm has the fastest convergence speed, a smooth curve, and the smallest training loss. For the German dataset, the adaptive optimization methods perform better. The initial progress of the Adam and AdaGrad algorithm is faster, while the convergence speed of the SGD algorithm and Momentum algorithm is slower, and the training loss is larger. For the Japanese dataset, the Adam algorithm has the fastest convergence speed and less training loss. The Momentum algorithm and the SGD algorithm have a slower convergence speed, and the AdaBound algorithm and the AmsBound algorithm have faster convergence speed and smooth curve.

In Figure 10, the X-axis represents the convergence speed of the algorithm, and the Y-axis indicates the number of training. Figures 11a-11c show the performance of BRNN with different optimization algorithms under different datasets. Under the Australian dataset, the highest accuracy of the BRNN model optimized by the SGD algorithm is 0.8723 , and the BRNN model optimized by AmsBound and AdaBound algorithms performs better. Under the German dataset, the AdaBound algorithm has the highest AUC (0.8101), the SGD model has the highest accuracy (0.78), and the AdaBound algorithm has the highest F-score. For Japanese datasets, the BRNN model optimized by the SGD algorithm, AmsBound algorithm, and AdaBound algorithm has a better classification effect. Thus, the AdaBound algorithm combines the advantages of the Adam algorithm and the SGD algorithm; it has a fast convergence speed, is insensitive to hyperparameters, and has good convergence and generalization. The optimized BRNN model achieves more accurate and reliable classification results on three datasets. In Figure 11, the X-axis represents the algorithm type, the left Y-axis indicates the optimization algorithm, and the right $\mathrm{Y}$-axis denotes the performance of different optimization algorithms on different datasets.

In Figure 12, the lower $\mathrm{X}$-axis represents the algorithm type, the upper $\mathrm{X}$-axis denotes the model performance, and the Y-axis indicates the optimization algorithm. Figures 12a-12c show the performance of the integrated BRNN + LR + XGBoost model optimized by different bionic optimization algorithms on three datasets. Obviously, the F-scores of the integrated deep learning model optimized by SGD, AmsBound, and AdaBound algorithms are better. Overall, in each dataset experiment, the performance of the proposed deep learning model is the best. Besides, under the same optimization algorithm, the classification effect of the integrated model BRNN + LR + XGBoost is significantly better than that of a single BRNN, proving that the integrated learning can improve the classification accuracy and model performance.

\section{Application of Integrated Deep Learning Model}

Figures A-C illustrate the performance results of different algorithm models under three different datasets. The financial risk management model in the latest research works are selected, in which CF-GA-Ens and EBCA-PSO-RF + XGBoost models are integrated models based on the original single models without using the deep learning method. Apparently, under three different datasets, compared with models without a deep learning method, the performance of the proposed integrated deep learning model is significantly improved, and the integrated model optimized by the AdaBound algorithm has achieved better classification results. The average AUC, the accuracy, and the F-score 
of the proposed integrated deep learning model have been increased by $3.1 \%, 2.3 \%$, and $2.2 \%$, respectively. Hence, deep learning improves the performance of the proposed model. Although the learning rate has been limited by the AdaBound algorithm, the dynamic boundary can avoid extreme values, thereby improving the model performance. In Figure 13, X-axis represents the type of model, and $\mathrm{Y}$-axis indicates the performance of different models under different datasets.

Figure 11a. Performance of BRNN under different optimization algorithms

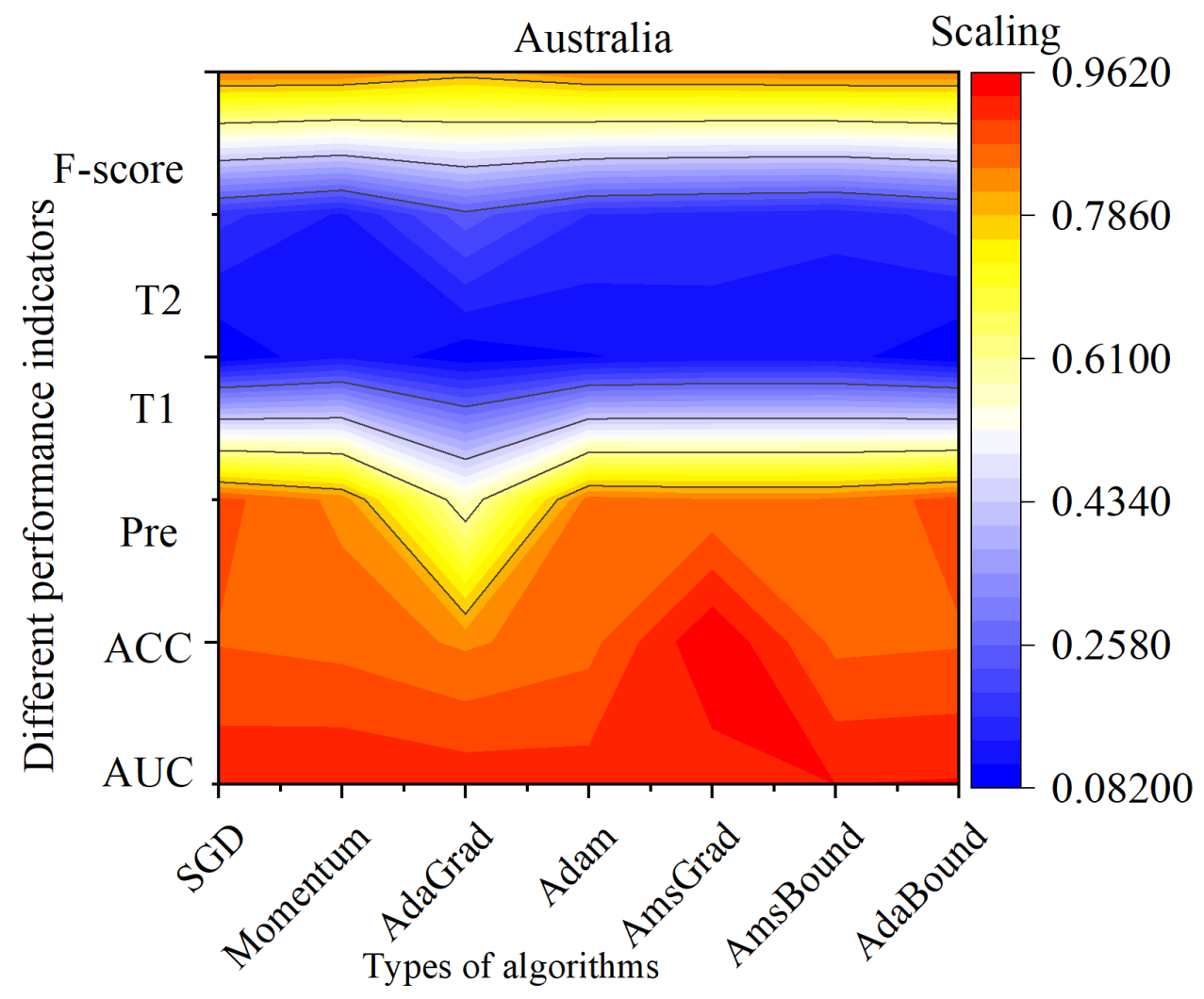


Figure 11b. Performance of BRNN under different optimization algorithms

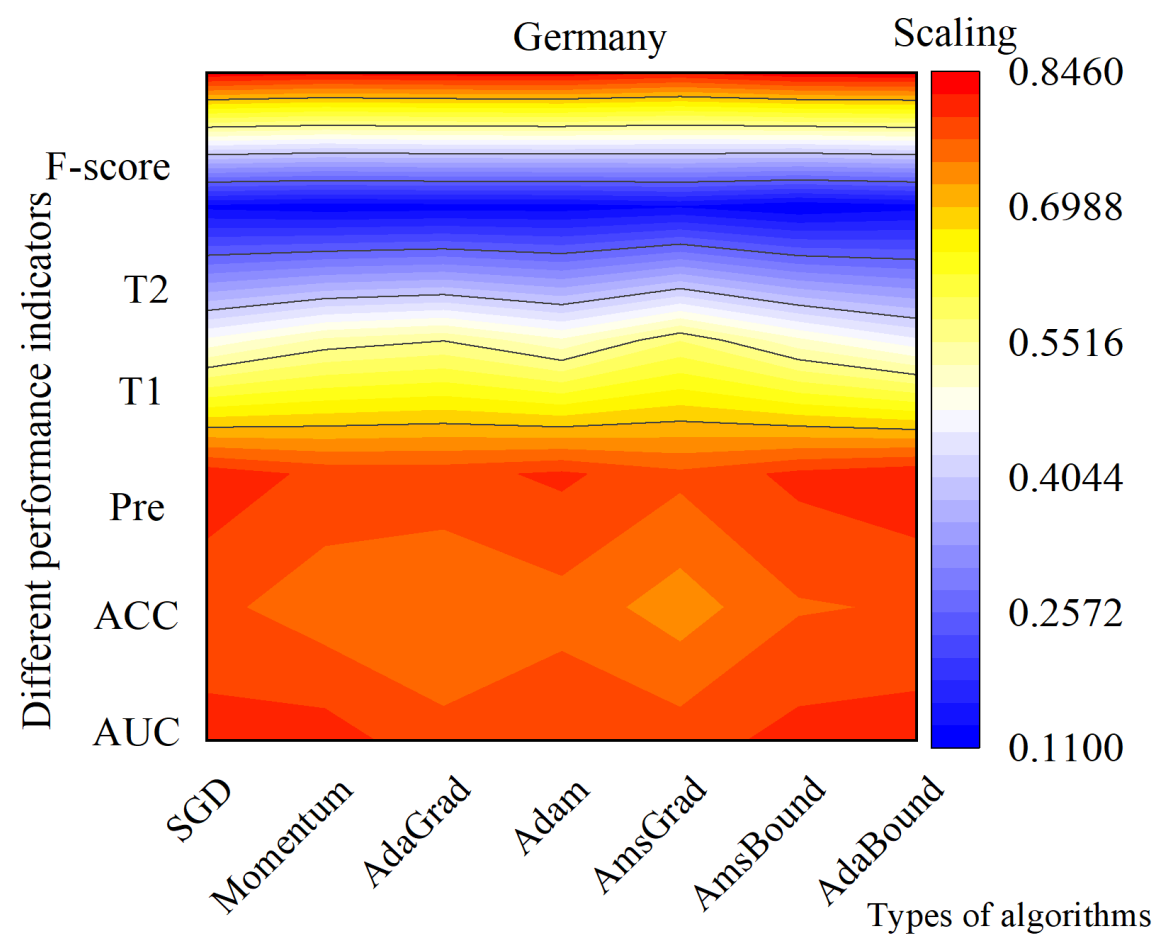

Figure 11c. Performance of BRNN under different optimization algorithms

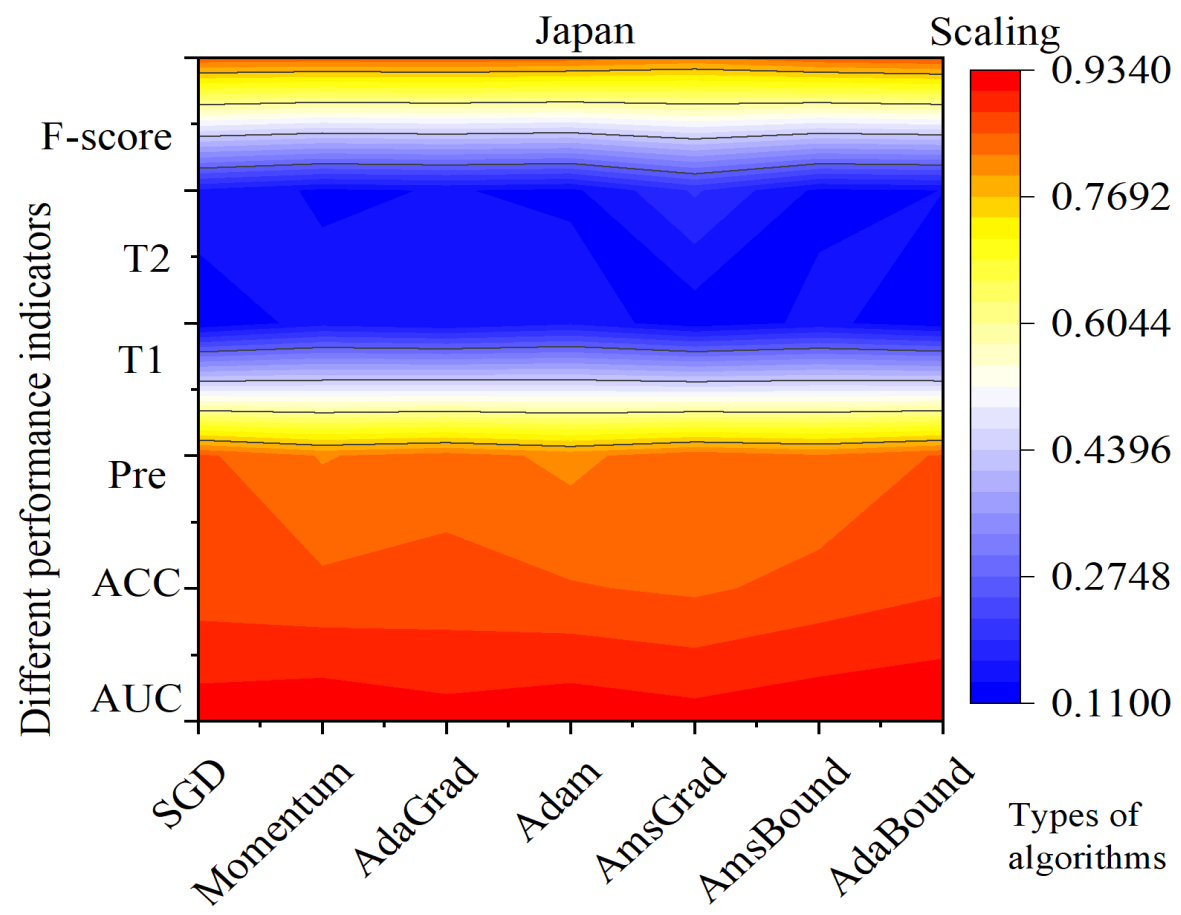


Figure 12a. Performance of the integrated $B R N N+L R+X G B o o s t$ under different optimization algorithms

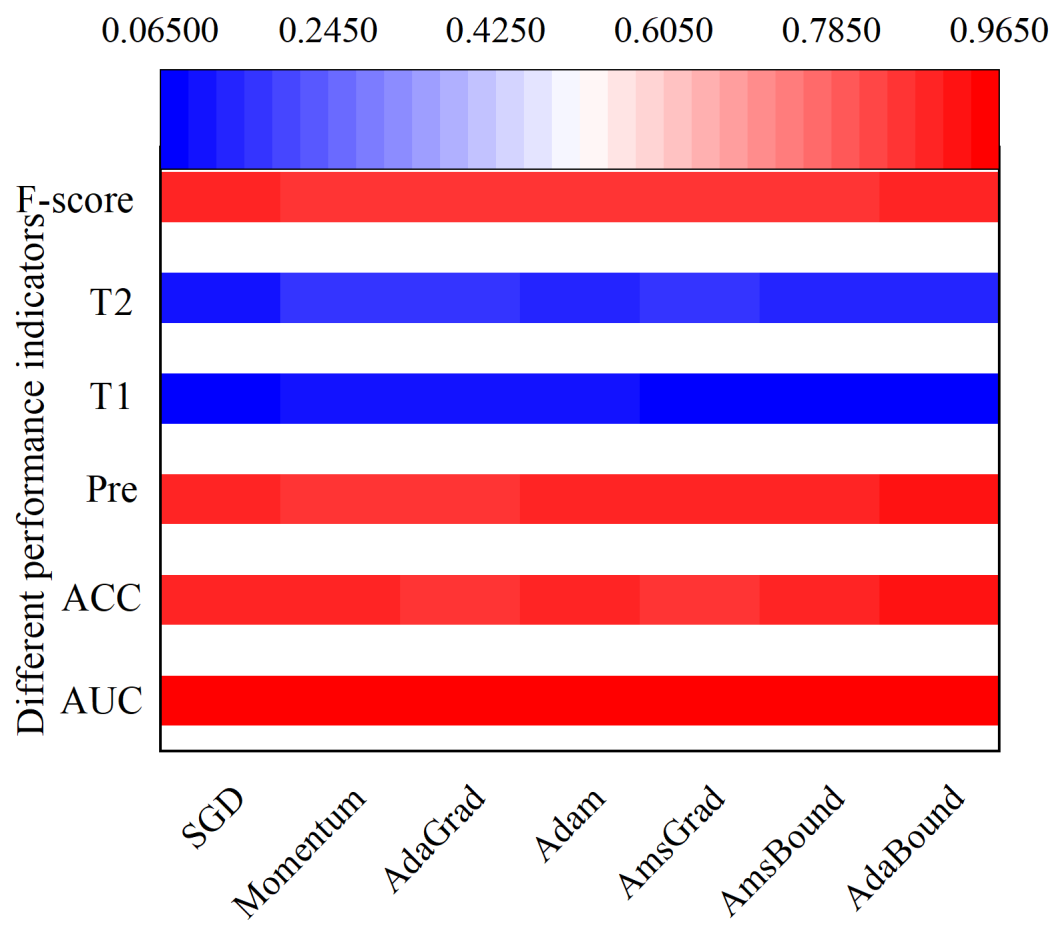

Figure 12b. Performance of the integrated BRNN $+L R+X G B o o s t$ under different optimization algorithms

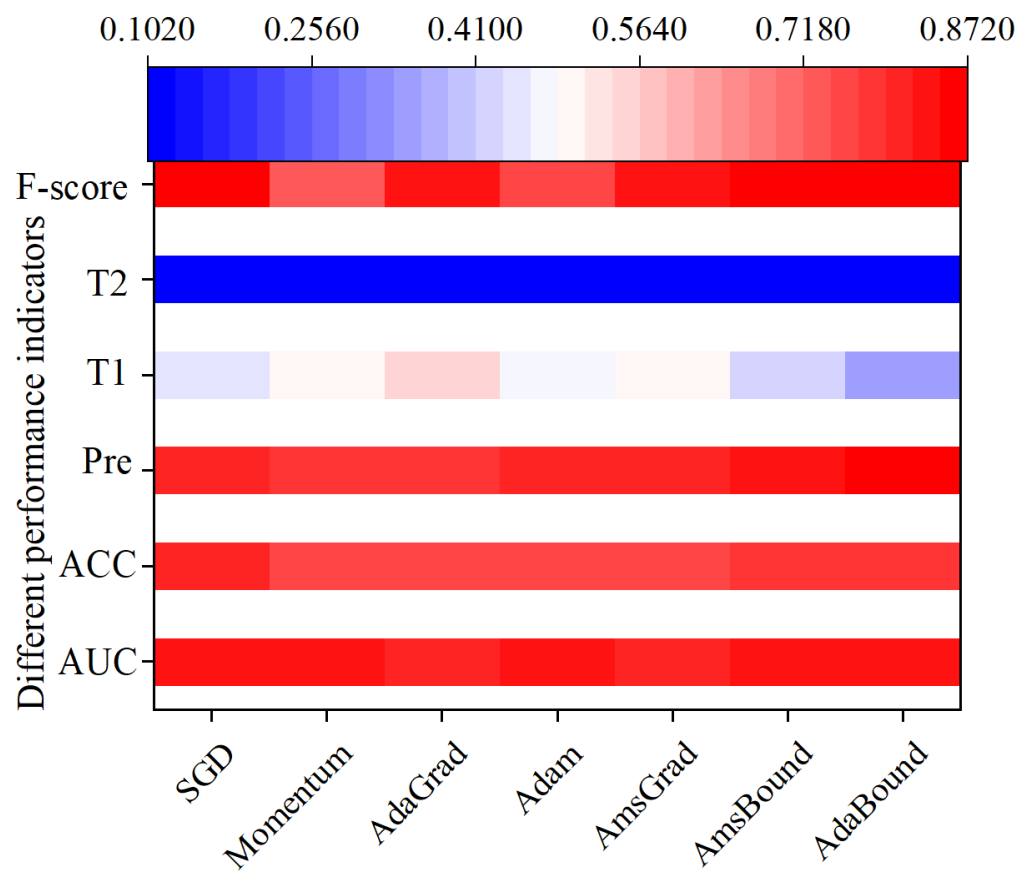


Figure 12c. Performance of the integrated $B R N N+L R+X G B o o s t$ under different optimization algorithms

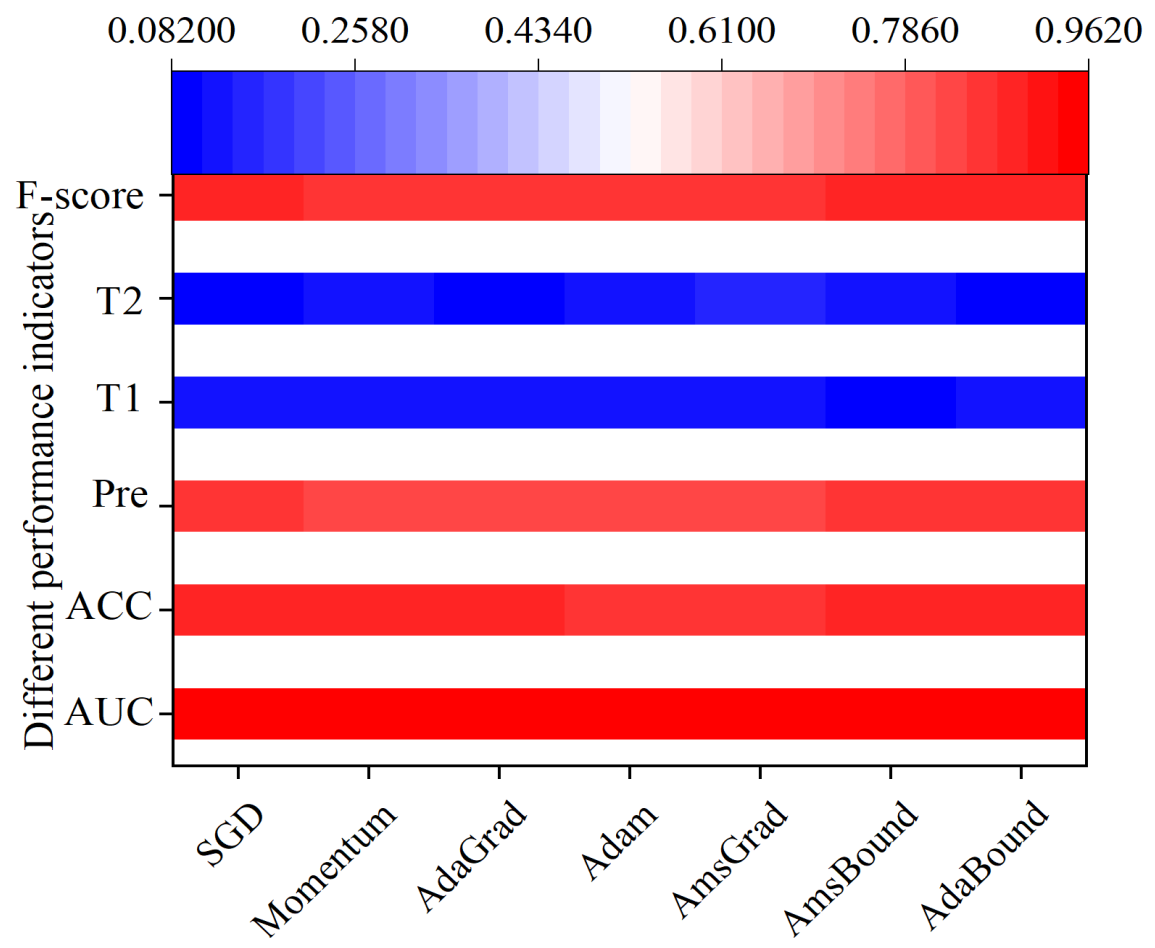

Figure 13a.

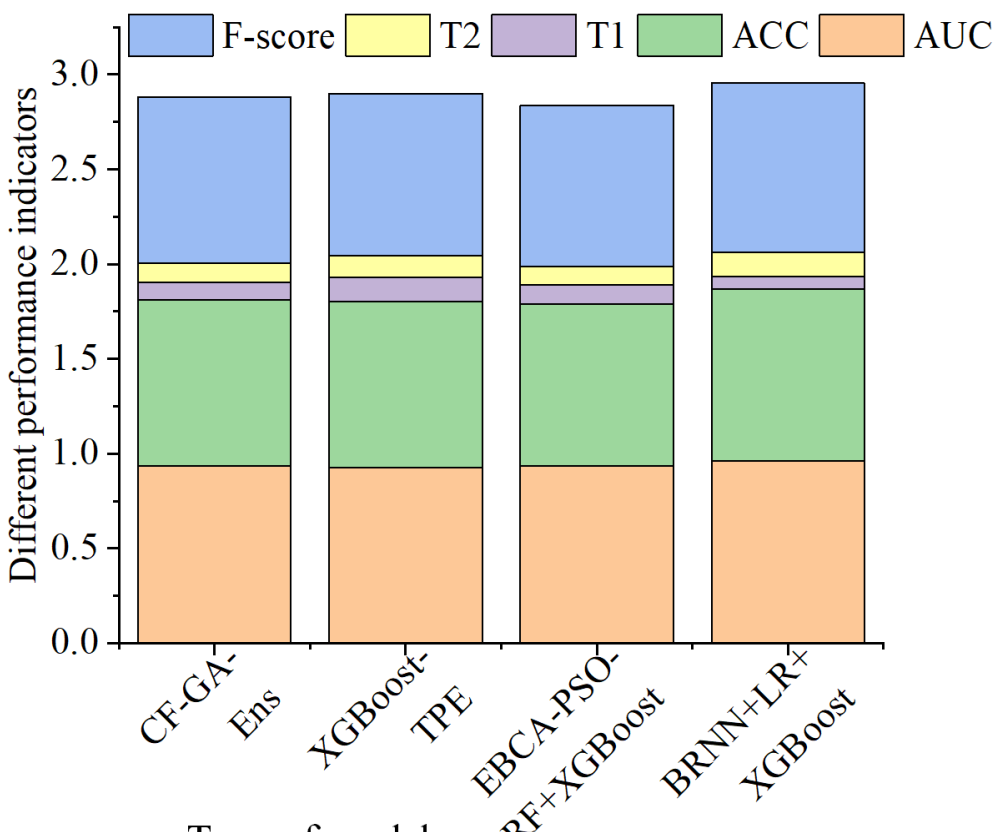

Type of model 
Figure 13b.

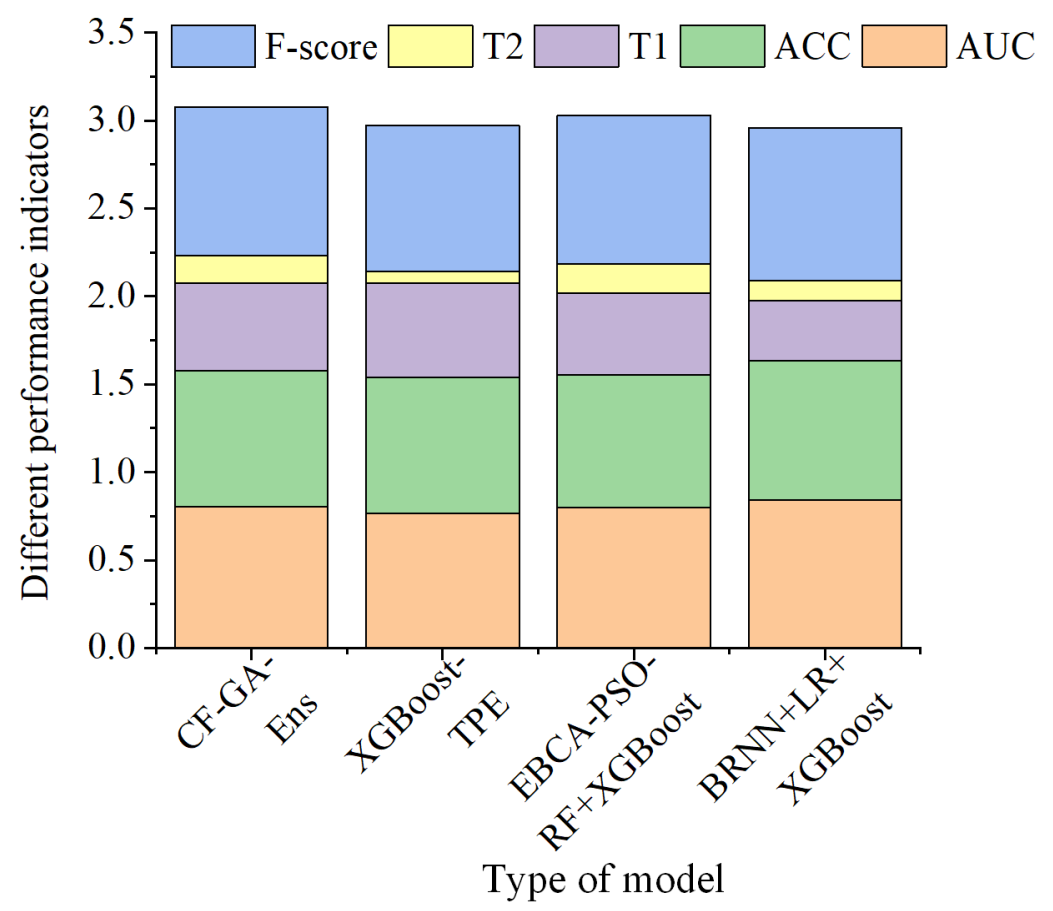

Figure 13c.

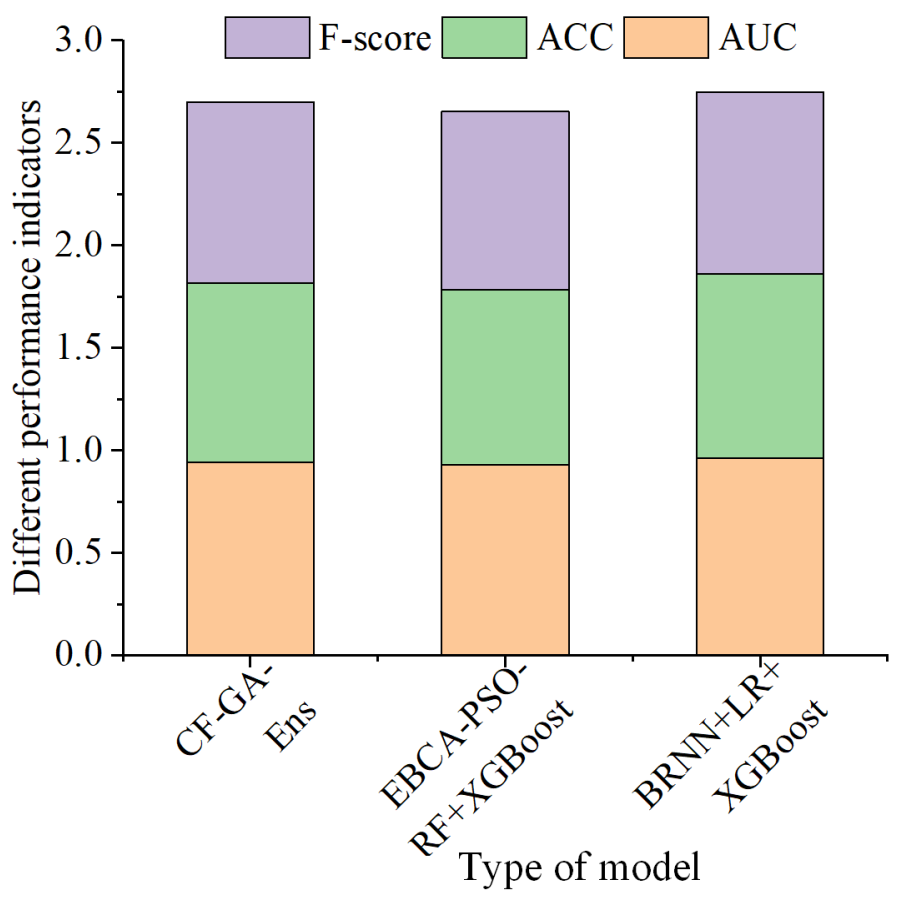




\section{DISCUSSION}

Therefore, the AdaBound algorithm introduced in this experiment has fast initialization progress, fast convergence speed, and excellent generalization. Specifically, it is similar to the adaptive optimization method at the early training stage. The experimental results of the performance index of different integrated classification models on different datasets show that integrated learning can improve classification accuracy and model generalization. For Australian, German, and Japanese datasets, the AdaBound algorithm combines the advantages of the Adam algorithm and the SGD algorithm and shows fast convergence, insensitivity to hyperparameters, and good generalization. The optimized BRNN model has achieved more accurate and reliable classification results on the three datasets. Under the same optimization algorithm, the classification effect of integrated model BRNN + LR + XGBoost is significantly better than a single BRNN model, thus proving that integrated learning can improve classification accuracy and model performance. Under three different datasets, compared with the model without a deep learning method, the performance of the proposed integrated deep learning model is significantly improved, and the integrated model optimized by the AdaBound algorithm achieves better classification results.

\section{CONCLUSION}

Based on the analysis of the existing financial market problems, the deep learning BRNN model is introduced to solve the insufficient credit scoring in the financial market. Then, the BRNN model is optimized by the bionic PSO algorithm and the AdaBound algorithm. Afterward, an integrated deep learning model is proposed based on the BRNN algorithm, bionic PSO algorithm, and AdaBound algorithm. Consequently, a financial market risk management system is constructed using the proposed integrated deep learning model, which provides a new reference for the related research of financial market risk management. The proposed model can converge faster and optimize different training stages through the bionic algorithm. Compared with the single deep learning model, the performance of the integrated deep learning model has greatly improved. Compared with the risk management system in the latest research, the accuracy of the constructed financial credit risk management system based on the integrated deep learning model is increased by $2.3 \%$, and the comprehensive evaluation results are also significantly improved. Although a risk management system is constructed for the financial market, there are still some shortcomings. Firstly, the financial data have not been pretreated with equalization. Besides, only the influence of hyperparameters is considered for model performance, while not all the parameters are optimized. In the future, big data analysis algorithms can be used to optimize all the parameters to solve the existing problems in financial market risk management. 


\section{REFERENCES}

Akbar, M., Akbar, A., \& Draz, M. U. (2021). Global Financial Crisis, Working Capital Management, and Firm Performance: Evidence From an Islamic Market Index. SAGE Open, 11(2). doi:10.1177/21582440211015705

Bachute, M. R., \& Subhedar, J. M. (2021). Autonomous Driving Architectures: Insights of Machine Learning and Deep Learning Algorithms. Machine Learning with Applications, 6, 1172. doi:10.1016/j.mlwa.2021.100164

Chen, M., Liu, Q., Huang, S., \& Dang, C. (2020). Environmental cost control system of manufacturing enterprises using artificial intelligence based on value chain of circular economy. Enterprise Information Systems, 1-20. Advance online publication. doi:10.1080/17517575.2020.1856422

Cox, T., \& Lowrie, K. (2021). From the Editors: Setting Risk Management Priorities. Risk Analysis, 41(8), 1255-1256. doi:10.1111/risa.13811

Crivello, J. C., Joubert, J. M., \& Sokolovska, N. (2021). Supervised deep learning prediction of the formation enthalpy of complex phases using a DFT database: The phase as an example. Computational Materials Science, 201. Advance online publication. doi:10.1016/j.commatsci.2021.110864

Dolfin, M., Leonida, L., \& Muzzupappa, E. (2019). Forecasting efficient risk/return frontier for equity risk with a KTAP approach-A case study in Milan Stock Exchange. Symmetry, 11(8), 1055-1063. doi:10.3390/ sym 11081055

Elena, F., Yolanda, H., \& Justo, P. (2019). New algorithmic framework for conditional value at risk: Application to stochastic fixed-charge transportation. European Journal of Operational Research, 277(1), $215-226$. doi:10.1016/j.ejor.2019.02.010

Flammer, C., \& Ioannou, I. (2021). Strategic management during the financial crisis: How firms adjust their strategic investments in response to credit market disruptions. Strategic Management Journal, 42(7), $1275-1298$. doi:10.1002/smj.3265

Guo, H., Zhou, J., \& Koopialipoor, M. (2019). Deep neural network and whale optimization algorithm to assess flyrock induced by blasting. Engineering with Computers, 1-14. doi:10.1007/s00366-019-00816-y

Hassib, E. M., El, D. A. I., \& Labib, L. M. (2020). WOA+ BRNN: An imbalanced big data classification framework using Whale optimization and deep neural network. Soft Computing, 24(8), 5573-5592. doi:10.1007/ s00500-019-03901-y

Hee, M. B., \& Christian, B. (2021). Systemic failures and organizational risk management in algorithmic trading: Normal accidents and high reliability in financial markets. Social Studies of Science, 51. doi:10.1177/03063127211048515 PMID:34612758

Kassi, D. F., Rathnayake, D. N., Louembe, P. A., \& Ding, N. (2019). Market risk and financial performance of non-financial companies listed on the Moroccan stock exchange. Risks, 7(1), 20-26. doi:10.3390/risks7010020

Liang, D. Y., Chen, Q. H., \& Liu, Y. L. (2021). Gated multi-attention representation in reinforcement learning. Knowledge-Based Systems, 233, 751. doi:10.1016/j.knosys.2021.107535

Licia, S. (2021). Corporate Financial Risk Management Interventions in the Organic Agri-Food Chains. Journal of Economics and Technology Research, 2(2), 764. doi:10.22158/jetr.v2n2p22

Liu, Y., Zhang, S., Chen, M., Wu, Y., \& Chen, Z. (2021). The Sustainable Development of Financial Topic Detection and Trend Prediction by Data Mining. Sustainability, 13(14), 7585. doi:10.3390/su13147585

Ma, X., Yang, R., Zou, D., \& Liu, R. (2020). Measuring the extreme risk of the sustainable financial system using GJR-GARCH model trading data-based. International Journal of Information Management, 50, 50526-50537. doi:10.1016/j.ijinfomgt.2018.12.013

Nikulin, E. E., \& Pekhterev, A. A. (2021). Turbulence on Financial Markets and a Model of a Multiplicative Cascade of Volatility. Mathematical Models and Computer Simulations, 13(4), 660-666. doi:10.1134/ S2070048221040177

Nosan, N. (2019). Retrospective Analysis of the Main Indicators of Ukraine's Financial Security. Modern Economics, 14(1), 193-199. doi:10.31521/modecon.V14(2019)-30 
Olivier, N., \& Lieven, D. M. (2019). Debt rating downgrades of financial institutions: Causality tests on singleissue CDS and iTraxx. Quantitative Finance, 19(12), 1975-1993. doi:10.1080/14697688.2019.1619933

Ouyang, Z. S., Yang, X. T., \& Lai, Y. Z. (2021). Systemic financial risk early warning of financial market in China using Attention-LSTM model. The North American Journal of Economics and Finance, $56,1917$. doi:10.1016/j.najef.2021.101383

Pang, G. Q., He, J., Huang, Y. M., \& Zhang, L. (2019). A Binary Logistic Regression Model for Severe Convective Weather with Numerical Model Data. Advances in Meteorology, 2019, 1-15. doi:10.1155/2019/6127281

Qian, X. Y., \& Olsen, T. L. (2020). Financial and risk management in agricultural cooperatives with application to the milk industry in New Zealand. International Journal of Production Research, 62, 1-31. doi:10.1080/00 207543.2020.1797204

Qiu, W. W. (2021). Enterprise financial risk management platform based on 5 G mobile communication and embedded system. Microprocessors and Microsystems, 80, 103594. doi:10.1016/j.micpro.2020.103594

Quezada-Téllez, L. A., Fernández-Anaya, G., Brun-Battistini, D., Nuñez-Zavala, B., \& Macías-Díaz, J. E. (2021). An Economic Model for OECD Economies with Truncated M-Derivatives: Exact Solutions and Simulations. Mathematics, 9(15), 1780. doi:10.3390/math9151780

Sha, Z., Hu, L., \& Rouyendegh, B. D. (2020). Deep learning and optimization algorithms for automatic breast cancer detection. International Journal of Imaging Systems and Technology, 30(2), 495-506. doi:10.1002/ ima. 22400

Shen, C., Min, C., \& Wang, C. (2019). Analyzing the trend of O2O commerce by bilingual text mining on social media. Computers in Human Behavior, 101, 474-483. doi:10.1016/j.chb.2018.09.031

Shi, Z., Wu, D., \& Guo, C. (2020). FCM-RDpA: TSK Fuzzy Regression Model Construction Using Fuzzy C-Means Clustering, Regularization, DropRule, and Powerball AdaBelief. 10.1016/j.ins.2021.05.084

Soares, C., Figueiredo, A., \& Figueiredo, F. (2021). The Subprime Crisis and Its Influence on Public, Private and Financial Sectors in European Countries. Journal of East-West Business, 27(1), 92-108. doi:10.1080/106 69868.2020.1830913

Soloschenko, M., \& Weber, E. (2021). Trend-Cycle Interactions and the Subprime Crisis: Analysis of US and Canadian Output. Journal of Business Cycle Research, 1-20. 10.1007/s41549-021-00058-2

Sun, T. S. (2021). Research on Financial Market Risk Based on GARCH-M Model. E3S Web of Conferences, 251. 10.1051/e3sconf/202125101106

Sun, Y. P., \& Li, Y. (2021). COVID-19 Outbreak and Financial Performance of Chinese Listed Firms: Evidence From Corporate Culture and Corporate Social Responsibility. Frontiers in Public Health, 9, 710743-710743. doi:10.3389/fpubh.2021.710743 PMID:34604155

Tang, W. G., Jiang, H., \& Zhang, Q. (2021). One-Bit Gridless DOA Estimation with Multiple Measurements Exploiting Accelerated Proximal Gradient Algorithm. Circuits, Systems, and Signal Processing, 40, 1-15. doi:10.1007/s00034-021-01829-z

Tiwari, I., Cruz, J. M., \& Parmananda, P. (2021). Stochastic Resonance Via Parametric Adaptation: Experiments and Numerics. Meeting Abstracts, 1(48), 261. doi:10.1149/MA2021-01481953mtgabs

Valeriane, J., \& Wolfgang, M. S. (2020). Measuring model risk in financial risk management and pricing. International Journal of Theoretical and Applied Finance, 23(02), 37. doi:10.1142/S0219024920500120

Veryzhenko, I. (2021). Who gains and who loses on stock markets? Risk preferences and timing matter. Intelligent Systems in Accounting, Finance \& Management, 28(2), 143-155. doi:10.1002/isaf.1493

Viktoriya, P., Svitlana, M., \& Oleksiy, B. (2019). Management of financial risks in the security system of enterprise development: economic and legal aspects. SHS Web of Conferences, 67. doi:10.1051/shsconf/20196706043

Yang, R., Liu, H., Nikitas, N., Duan, Z., Li, Y., \& Li, Y. (2021). Short-term wind speed forecasting using deep reinforcement learning with improved multiple error correction approach. Energy, 239, 168. doi:10.1016/j. energy.2021.122128 
Yang, R., Yu, L., Zhao, Y., Yu, H., Xu, G., Wu, Y., \& Liu, Z. (2020). Big data analytics for financial market volatility forecast based on support vector machine. International Journal of Information Management, 50, 50452-50462. doi:10.1016/j.ijinfomgt.2019.05.027

Zhou, P., Yuan, X. T., Lin, Z. C., \& Hoi, S. (2021). A Hybrid Stochastic-Deterministic Minibatch Proximal Gradient Method for Efficient Optimization and Generalization. IEEE Transactions on Pattern Analysis and Machine Intelligence, 56, 159. doi:10.1109/TPAMI.2021.3087328 PMID:34101583

Zhou, Y. (2021). Systemic Financial Risk Prediction Using Deep Spatio-Temporal Neural Network. Frontiers in Economics and Management, 2(4). Advance online publication. doi:10.6981/FEM.202104_2(4).0018

Peng Du was born in Weinan, Shaanxim China, in 1980. He received the Bachelor's and Master's degrees from Northwest University of Political Science and Law, P.R. China. Now, he works in School of Economics and Management, Shaanxi University of Science and Technology, His research interests include corporate law, financial law, corporate governance and information security. Peng Du is affiliated with the School of Economics and Management, Shaanxi University of Science and Technology, Xi'an, 710021, China Peng Du is the corresponding author.E-mail: 24451038@qq.com.

Hong Shu was born in Weinan, Shaanxi, China, in 1995. She received Master's degrees from Chang'an University of China. Now, she is now a in-service teacher in Department of Public Courses, Shaanxi Polytechnic Institute of China. Her research interests include probability theory and mathematical statistics, algorithm design, etc. 